\title{
Targeting PLK1 overcomes T-DM1 resistance via CDK1-dependent phosphorylation and inactivation of Bcl-2/xL in HER2-positive breast cancer
}

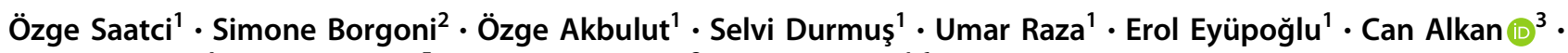 \\ Aytekin Akyol $^{4}$ Özgür Kütük ${ }^{5} \cdot$ Stefan Wiemann ${ }^{2} \cdot$ Özgür Şahin ${ }^{1,6}$
}

Received: 7 May 2017 / Revised: 22 October 2017 / Accepted: 12 November 2017 / Published online: 2 February 2018

(c) Macmillan Publishers Limited, part of Springer Nature 2018

\begin{abstract}
Trastuzumab-refractory, HER2 (human epidermal growth factor receptor 2)-positive breast cancer is commonly treated with trastuzumab emtansine (T-DM1), an antibody-drug conjugate of trastuzumab and the microtubule-targeting agent, DM1. However, drug response reduces greatly over time due to acquisition of resistance whose molecular mechanisms are mostly unknown. Here, we uncovered a novel mechanism of resistance against T-DM1 by combining whole transcriptome sequencing (RNA-Seq), proteomics and a targeted small interfering RNA (siRNA) sensitization screen for molecular level analysis of acquired and de novo T-DM1-resistant models of HER2-overexpressing breast cancer. We identified Polo-like kinase 1 (PLK1), a mitotic kinase, as a resistance mediator whose genomic as well as pharmacological inhibition restored drug sensitivity. Both acquired and de novo resistant models exhibited synergistic growth inhibition upon combination of TDM1 with a selective PLK1 inhibitor, volasertib, at a wide concentration range of the two drugs. Mechanistically, T-DM1 sensitization upon PLK1 inhibition with volasertib was initiated by a spindle assembly checkpoint (SAC)-dependent mitotic arrest, leading to caspase activation, followed by DNA damage through CDK1-dependent phosphorylation and inactivation of Bcl-2/xL. Furthermore, we showed that Ser70 phosphorylation of Bcl-2 directly regulates apoptosis by disrupting the binding to and sequestration of the pro-apoptotic protein Bim. Importantly, T-DM1 resistance signature or PLK1 expression correlated with cell cycle progression and DNA repair, and predicted a lower sensitivity to taxane/trastuzumab combination in HER2-positive breast cancer patients. Finally, volasertib in combination with T-DM1 greatly synergized in models of TDM1 resistance in terms of growth inhibition both in three dimensional (3D) cell culture and in vivo. Altogether, our results provide promising pre-clinical evidence for potential testing of T-DM1/volasertib combination in T-DM1 refractory HER2positive breast cancer patients for whom there is currently no treatment available.
\end{abstract}

Electronic supplementary material The online version of this article (https://doi.org/10.1038/s41388-017-0108-9) contains supplementary material, which is available to authorized users.

\footnotetext{
Özgür Şahin

sahinozgur@gmail.com

1 Department of Molecular Biology and Genetics, Faculty of Science, Bilkent University, 06800 Ankara, Turkey

2 Division of Molecular Genome Analysis, German Cancer Research Center (DKFZ), INF580, Heidelberg 69120, Germany

3 Department of Computer Engineering, Bilkent University, 06800
}

\section{Introduction}

Breast cancer is the second most commonly diagnosed cancer among women, and despite the recent improvements in diagnosis and treatment of the disease, it is still one of the leading causes of death worldwide [1]. Twenty to thirty percent of breast cancers are characterized by the amplification/overexpression of ERBB2/HER2 (human epidermal

Ankara, Turkey

4 Department of Pathology, Hacettepe University School of Medicine, 06410 Ankara, Turkey

5 Department of Medical Genetics, Başkent University, 01250 Adana, Turkey

6 National Nanotechnology Research Center (UNAM), Bilkent University, 06800 Ankara, Turkey 
growth factor receptor 2), which is associated with poor prognosis, aggressive disease characteristics and resistance to chemotherapy $[2,3]$. In the past 20 years, cancer research has led to the development of highly promising therapeutic agents that mostly target cancer driver proteins and thus antagonize tumor growth and increase survival, also in highly heterogeneous breast cancer [4]. Trastuzumab (Herceptin ${ }^{\circledR}$ ) is the first therapeutic humanized monoclonal antibody and was approved by the Food and Drug Administration (FDA) in 1998 for the treatment of HER2positive metastatic breast cancer (MBC) [5]. It binds the extracellular domain IV of HER2 and prevents the activation of its intracellular tyrosine kinase domain [6]. Hence, it prevents activation of MAPK and PI3K/Akt pathways, which, in turn, leads to G1 arrest as well as suppression of cell growth and proliferation [7, 8]. Despite the initial clinical benefit achieved by trastuzumab, around $70 \%$ of trastuzumab-treated patients have either primary (de novo) or secondary (acquired) resistance [9].

Detailed identification of the resistance mechanisms against trastuzumab together with recent advances in genetic engineering have led to the development of secondline therapeutic agents including antibody-drug conjugates (ADCs) [10]. Trastuzumab emtansine (T-DM1, Kadcyla ${ }^{\circledR}$ ) is a next-generation HER2-ADC, combining trastuzumab, along with its cytostatic functions, with a potent microtubule-targeting agent, DM1 (derivative of maytansine 1) via a stable linker, MCC $[11,12]$. Once the HER2 and T-DM1 complex is internalized upon binding, DM1 is released to the cytoplasm where it disrupts microtubule assembly, thus causing cell cycle arrest at G2/M and subsequent apoptotic cell death [13, 14]. T-DM1 has shown greater anti-tumor activity as compared to non-conjugated trastuzumab, even in trastuzumab-refractory HER2-amplified cell line and xenograft models [12]. In 2012, a key clinical trial (EMILIA) reported the efficacy and safety of TDM1 for HER2-positive advanced or MBC patients who were previously treated with trastuzumab and a taxane. The median progression-free survival was 9.6 months with TDM1 compared to 6.4 months with the lapatinib and capecitabine combination. Moreover, T-DM1 treatment increased the overall survival by around 6 months $[11,12]$. Based on these favorable efficacy results, T-DM1 was swiftly approved by FDA in 2013. Even though it offers a huge clinical benefit for previously treated HER2-positive breast cancer patients, a substantial number of patients eventually progress and frequently develop acquired resistance [14]. Therefore, understanding the mechanisms of TDM1 resistance is crucial for the treatment of HER2positive breast cancer and could establish new targets for therapeutic intervention.

In this study, we uncovered a novel mechanism of $\mathrm{T}$ DM1 resistance in HER2-positive breast cancer. Whole transcriptome sequencing of sensitive and resistant cell line models deciphered differentially expressed genes, and a targeted small interfering RNA (siRNA) sensitization screen identified Polo-like kinase 1 (PLK1) as an important mediator of resistance. PLK1 inhibition by volasertib (BI 6727) induced a strong spindle assembly checkpoint (SAC)dependent mitotic arrest, leading to apoptosis and caspasedependent DNA damage via CDK1-dependent phosphorylation and inactivation of $\mathrm{Bcl}-2 / \mathrm{Bcl}-\mathrm{xL}$. Combination treatment of acquired and de novo resistant models with $\mathrm{T}$ DM1 and volasertib resulted in synergistic growth inhibition over a wide dose range of the two drugs. Correlation analysis within the mRNA and protein expression data of breast cancer patients revealed a strong association of the TDM1 resistance signature (T-DM1R signature) with cell cycle progression/DNA repair and a worse response to taxane/trastuzumab combination in HER2-positive breast cancer. Finally, the strong synergy between T-DM1 and volasertib in attenuating cell growth in three-dimensional (3D) culture and the growth of tumors in vivo provided preclinical support for the role of PLK1 inhibition in T-DM1 sensitization.

\section{Results}

\section{Acquired T-DM1 resistance is characterized by loss of T-DM1-induced mitotic arrest, apoptosis and DNA damage in HER2-positive breast cancer}

In order to characterize mechanisms of T-DM1 resistance and to identify the molecular mediators of the resistanceassociated phenotypes in HER2-positive breast cancer, we developed two different T-DM1-resistant models in vitro, BT-474 T-DM1R and SK-BR-3 T-DM1R. To this end, we have continuously treated the parental cells with T-DM1 over 9 months, starting from IC25 and by gradually increasing the dose until a significant difference between the T-DM1 response of the wild-type (WT) and resistant cells has been achieved (Fig. 1a). As a result, we obtained two different resistant models: BT-474 T-DM1R, being resistant to $4 \mu \mathrm{g} / \mathrm{ml}$ of T-DM1, and SK-BR-3 T-DM1R, being resistant to $0.05 \mu \mathrm{g} / \mathrm{ml}$ of $\mathrm{T}-\mathrm{DM} 1$. Interestingly, both the parental and resistant counterparts of SK-BR-3 cell line were considerably more sensitive to T-DM1, as compared to the parental and resistant counterparts of BT-474. This could be due to the fact that the two cell lines belong to two different clinical subtypes of breast cancer: BT-474 belongs to luminal B subtype, whereas SK-BR-3 belongs to HER2positive subtype. Therefore, they might exhibit heterogeneity in terms of their response to T-DM1 treatment, which may further explain the difference between the IC50 values of BT-474 and SK-BR-3 T-DM1R cells $(20.25 \mu \mathrm{g} /$ 
$\mathrm{ml}$ for BT-474, compared to $0.055 \mu \mathrm{g} / \mathrm{ml}$ for SK-BR-3 TDM1R) [15-17]. Few studies have reported G2/M arrest followed by apoptosis as a mechanism of T-DM1-induced cytotoxicity $[18,19]$. In line with this, we observed a prominent increase in the percentage of G2/M-arrested cells in sensitive models upon treatment with the dose of T-DM1 to which T-DM1R models are resistant ( 4 and $0.05 \mu \mathrm{g} / \mathrm{ml}$ for BT-474 and SK-BR-3, respectively). Such an arrest at the G2/M phase of the cell cycle was completely absent in TDM1-resistant counterparts (Fig. 1b). In order to further characterize the cell cycle-associated changes upon T-DM1 treatment of the WT and T-DM1R models in detail, we performed Reverse Phase Protein Array (RPPA) analysis to quantify cell cycle regulators, checkpoint proteins and their targets (Fig. 1c). (The list of antibodies used for RPPAs is given in Supplementary Table S1.) Among the screened proteins, cyclin B1 and phosphorylated histone H3 at Ser10 (p-Histone H3 (Ser10)), both of which are markers of $\mathrm{M}$ phase, demonstrated a highly consistent pattern in both cell line models, as they were upregulated in a dose-dependent manner exclusively in WT cells. Intriguingly, phosphorylation of two of the DNA damage checkpoint targets, histone $\mathrm{H} 2 \mathrm{AX}$ and $\mathrm{p} 53$, also showed a similar type of regulation, suggesting a previously unknown role of $\mathrm{T}$ DM1 in generating DNA breaks and inducing DNA damage response together with a prolonged mitosis. These results were validated by Western blot analysis of the mitotic arrest and DNA damage markers (Fig. 1d). Immunofluorescence staining of p-Histone $\mathrm{H} 3$ and $\mathrm{p}-\mathrm{H} 2 \mathrm{AX}$ further verified the increase in the number of WT cells arrested in mitosis and having damaged DNA upon T-DM1 treatment to a much higher extent than in T-DM1R models (Fig. 1e, f, respectively). Since T-DM1 exerts its cytotoxic functions mostly in cells undergoing mitosis, by interfering with normal mitotic progression; altered cell cycle kinetics, for example, an increased doubling time of the resistant models, might cause attenuation of T-DM1 response, and result in the observed resistance phenotype. To exclude this possibility, we compared the proliferation kinetics of WT and T-DM1R cells. As can be seen in Supplementary Figure S1, there was no significant difference between the doubling time of WT and T-DM1R models, suggesting that altered cell cycle kinetic is unlikely associated with T-DM1 resistance.

Alterations in the level of surface HER2 expression, which might also lead to attenuation of the rate of HER2/ antibody complex internalization, have previously been associated with resistance against trastuzumab [20, 21]. To test whether such an alteration in the transport kinetics of TDM1 modulates drug resistance also in current models, we first examined the expression of HER2 in WT versus resistant cells. As can be seen in Supplementary Figure $\mathrm{S} 2 \mathrm{~A}$, there was no difference in the HER2 expression of WT and T-DM1R cells. Moreover, the internalization rate of T-DM1/HER2 complex in WT versus resistant models were highly similar (Supplementary Figure S2B), which indicates that acquired T-DM1 resistance is most probably governed by mechanisms that are not directly related to altered HER2 expression and/or internalization kinetics. Upon internalization of HER2/T-DM1 complex, DM1 induces microtubule depolymerization [22]. After we demonstrated similar T-DM1 internalization kinetics in WT and T-DM1R cells, as a next step, we analyzed the organization of microtubules in sensitive versus resistant models under T-DM1 treatment. Microtubule depolymerization has started at the IC50 dose in WT cells and gradually increased with increasing doses of T-DM1. Importantly, depolymerization of microtubules was also apparent in the resistant counterparts treated with the same doses of T-DM1 (Supplementary Figure S2C). Overall, these results suggest a similar internalization kinetics of T-DM1, which leads to microtubule depolymerization in both sensitive and resistant cells, although the resistant models override the mitotic arrest caused by abnormal spindle formation.

We further demonstrated a differential regulation of apoptosis in T-DM1 resistance, where the drastic increase in caspase-3/7 activity observed in WT cells upon T-DM1 treatment was completely abrogated in T-DM1R models (Fig. 1g). The lack of apoptosis in the resistant models was also evident in the Western blot analysis of the apoptotic markers, cleaved caspase-3 and cleaved poly(ADP-ribose) polymerase (PARP) (Fig. 1h). Strikingly, the high-level caspase activity upon T-DM1 treatment of the WT cells was greatly contributing to T-DM1-induced cell death, as combining a pan-caspase inhibitor, Q-VD-Oph, with T-DM1 significantly attenuated the inhibitory effect of the drug on cellular survival in drug-sensitive models (Fig. 1i). Taken together, acquired T-DM1 resistance was characterized by a loss of drug-induced mitotic arrest, apoptosis and DNA damage in HER2-positive breast cancer.

\section{Whole transcriptome analysis combined with a targeted siRNA sensitization screen identifies PLK1 as a mediator of T-DM1 resistance}

Having identified the processes involved in T-DM1 resistance in HER2-positive breast cancer at the phenotypic level, we sought to identify the molecular mediators of resistance which can be exploited as therapeutic targets to overcome resistance. For this purpose, we performed differential gene expression analysis in our WT and T-DM1R models of BT-474 and SK-BR-3 cell lines with RNA sequencing. As a result, 2000 and 3728 genes were found to be significantly differentially expressed between the WT and resistant models of BT-474 and SK-BR-3, respectively ( $p$ value $<0.05$ ), among which 331 mRNAs were common to both models (Fig. 2a, Supplementary Table S2). Among 
A
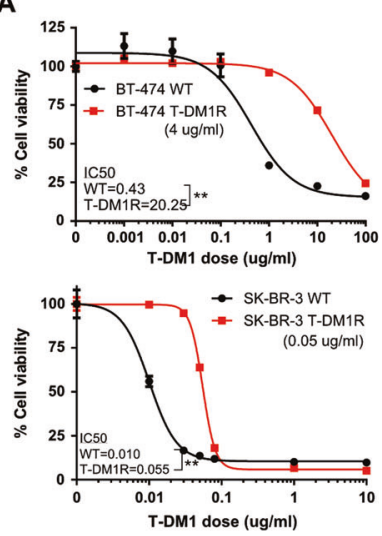

C

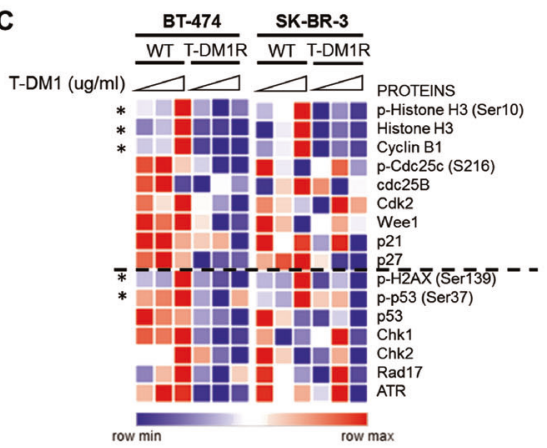

E

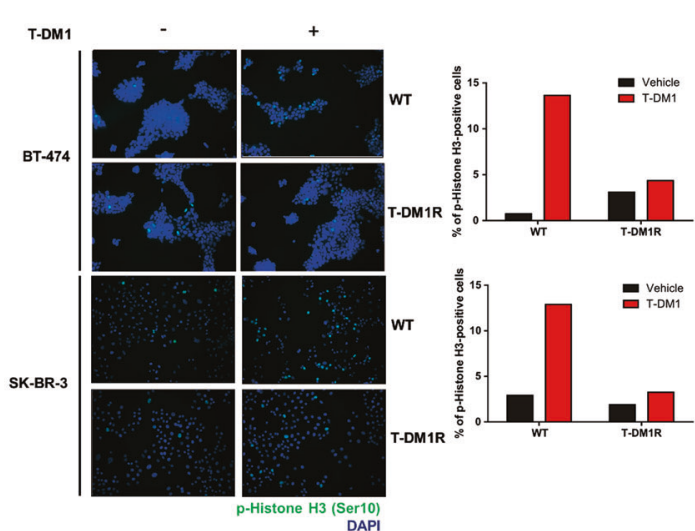

G
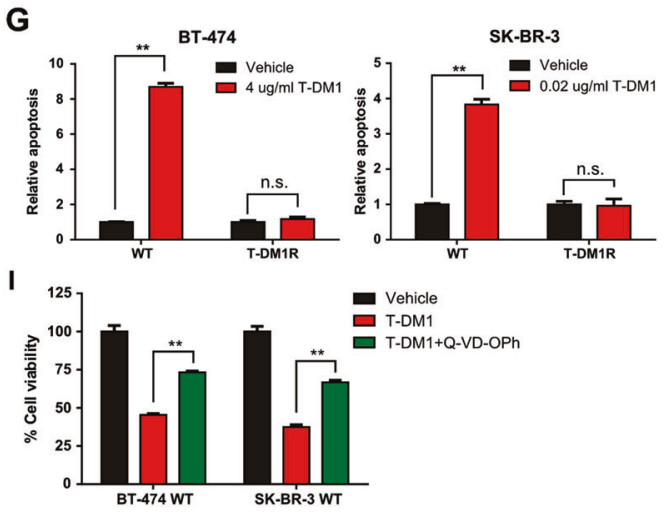

those 331 genes, we specifically focused on the ones upregulated in both of the resistant models, and further narrowed down the number of candidates to 20 by selecting

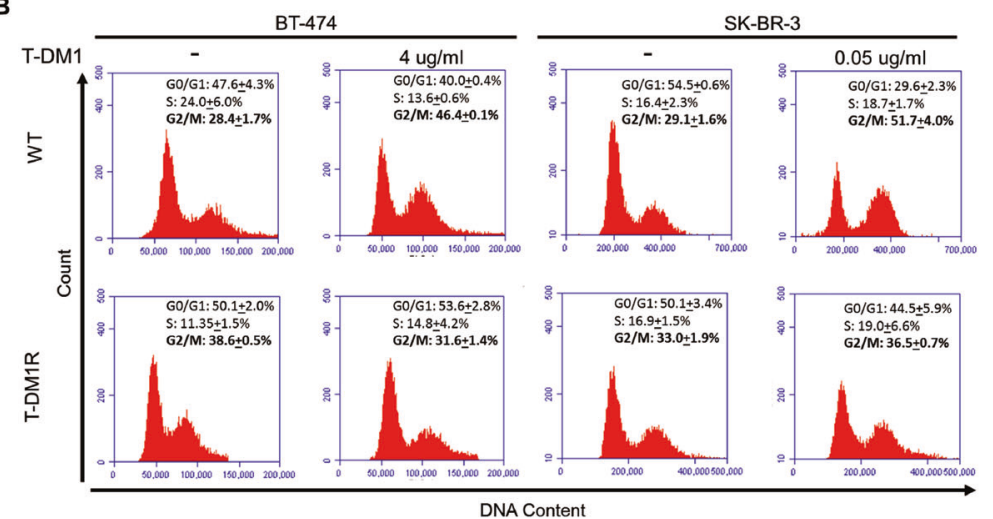

D

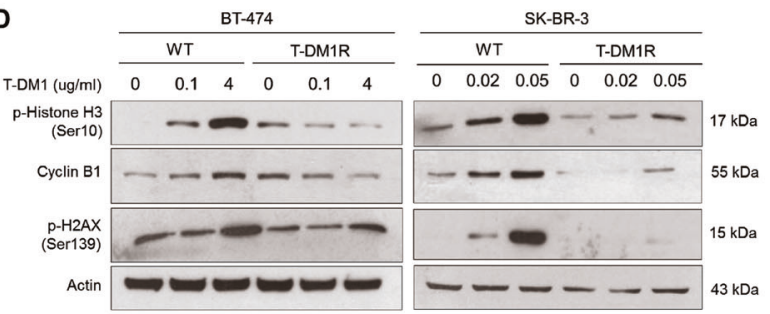

$F$

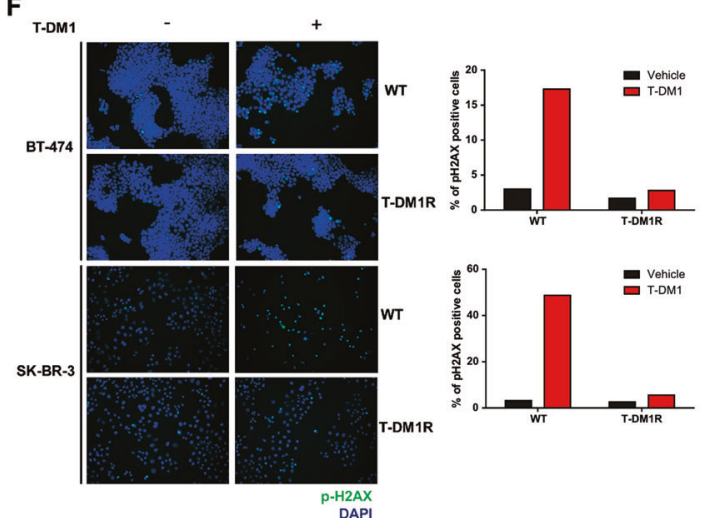

H

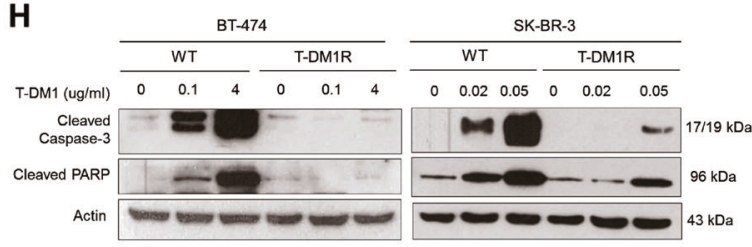

the upregulated genes that have druggable protein products (Fig. 2a). Expression of these 20 genes was then knocked down by specific siRNA sequences in a siRNA sensitization 
Fig. 1 Acquired T-DM1 resistance is characterized by loss of T-DM1induced mitotic arrest, apoptosis and DNA damage in HER2-positive breast cancer. a Dose-response curves of the WT and T-DM1R models, treated with increasing doses of T-DM1 for $72 \mathrm{~h}$. Cell viability was measured by CellTiter-Glo Kit as quadruplicates in all the following cell viability experiments, unless otherwise indicated. b 7AAD staining of the WT and T-DM1R models treated with T-DM1 (4 and $0.05 \mu \mathrm{g} / \mathrm{ml}$ for BT-474 and SK-BR-3, respectively) for $24 \mathrm{~h}$, showing a loss of G2/M arrest in the resistant models. $\mathbf{c}$ RPPA results for the cell cycle regulators, checkpoint proteins and their targets, differentially regulated upon $48 \mathrm{~h}$ treatment with increasing doses of TDM1 in WT and T-DM1R models $(0.1$ and $4 \mu \mathrm{g} / \mathrm{ml}$ for BT- 474 and 0.02 and $0.05 \mu \mathrm{g} / \mathrm{ml}$ for SK-BR-3). The proteins showing a consistent change in both of the models are highlighted with an asterisk. d Western blot validation of the RPPA data for the mitotic arrest and DNA damage markers. Actin was used as a loading control. e, f Immunofluorescence staining of p-Histone H3 (Ser10) (e) and pH2AX (Ser139) (f) in the WT and T-DM1R cells treated with 4 and $0.05 \mu \mathrm{g} / \mathrm{ml}$ of T-DM1 for BT-474 and SK-BR-3, respectively, for $24 \mathrm{~h}$. Alexa Fluor 488 secondary antibody was used for the visualization. The number of positively stained cells was provided for each cell line next to its image. A total of 300 cells were counted for each condition. g Relative apoptosis induced by 0.02 and $4 \mu \mathrm{g} / \mathrm{ml}$ of T-DM1 treatment for $72 \mathrm{~h}$ in the WT and T-DM1R models of BT-474 and SK-BR-3, respectively, based on the activity of caspase-3/7 with Caspase-Glo Assay Kit. Statistical significance was determined with an unpaired, two-tailed Student's $t$ test. h Western blot analysis of the apoptotic markers, cleaved caspase- 3 and PARP in the WT and T-DM1R models treated with 0.1 and $4 \mu \mathrm{g} / \mathrm{ml}$ for BT-474 and 0.02 and $0.05 \mu \mathrm{g} / \mathrm{ml}$ for SK-BR-3 for $48 \mathrm{~h}$. i Loss of T-DM1-induced growth inhibition with caspase inhibition by Q-VD-Oph, a pan-caspase inhibitor. Cells were pre-treated with $50 \mu \mathrm{M}$ of Q-VD-OPh, $1 \mathrm{~h}$ before $72 \mathrm{~h}$ treatment with 4 and $0.05 \mu \mathrm{g} / \mathrm{ml}$ of T-DM1, for BT-474 and SK-BR-3, respectively. Statistical significance between two groups was determined with an unpaired, two-tailed Student's $t$ test. $* * P<0.01$. This applies to all figures shown. NS not significant

screen, and the impact of gene knockdown on viability of TDM1-treated resistant cells was evaluated (Fig. 2b). Zscores for each knockdown were calculated based on the difference in viability of cells treated with T-DM1 alone or together with the respective siRNA. Among the 20 knockdowns performed, silencing Polo-like kinase 1 (PLK1) gave a $z$-score lower than -2 in both resistant models, indicating substantial level of sensitization (Fig. 2b). PLK1 is an important serine/threonine kinase mediating diverse functions in cell cycle, especially in the mitotic phase [23, 24]. The initial screen results for PLK1 were validated in an independent experiment using two different siRNA sequences against PLK1 in both resistant models (Fig. 2c). Overexpression of PLK1 in the resistant models and the PLK1 knockdown by siPLK1 treatment at both mRNA and protein level were validated with quantitative real-time polymerase chain reaction (qRT-PCR) and Western blot experiments, respectively (Supplementary Figure S3A-C). Since we observed a substantial loss of mitotic arrest upon T-DM1 treatment in resistant models (Fig. 1d), we hypothesized that overexpression of the mitotic kinase, PLK1, would be highly essential for execution of a faithful mitosis in T-DM1 resistance. To test this, we performed flow cytometric analysis of PLK1, together with the mitosis marker p-Histone H3 in WT and resistant cells under TDM1 treatment. As expected, there was a significant increase in the percentage of p-Histone H3-positive, that is, mitotic cells upon T-DM1 treatment in WT models, as compared to no arrest in their resistant counterparts (Supplementary Figure S3D, left panel). Importantly, mitotic cells of the resistant models, which undergo normal mitosis in the presence of T-DM1, were stained stronger for PLK1 as compared to their WT counterparts, with 54.8 and $62.4 \%$ of T-DM1R cells expressing higher levels of PLK1 than their WT counterparts (middle and right panels of Supplementary Figure S3D). PLK1 is a druggable target and a few inhibitors are currently tested in phase II/III trials [25]. Volasertib is an ATP-competitive inhibitor of PLK1, effective even at very low concentrations, and is currently tested in phase III trials for the treatment of previously untreated acute myeloid leukemia (AML) patients who are ineligible for intensive therapy [26, 27]. For the combination treatment, we have chosen the IC25 of both T-DM1 and volasertib, which are around 20 and $0.06 \mu \mathrm{g} / \mathrm{ml} \mathrm{T-DM1}$ and 25 and $15 \mathrm{nM}$ volasertib for BT-474 and SK-BR-3 TDM1R, respectively. Our results demonstrated that combination of T-DM1 with volasertib caused a stronger growth inhibition than what was conferred by individual drugs in both T-DM1R models, thus validating the effects of siRNA knockdown of PLK1 on T-DM1 sensitivity (Fig. 2d). Furthermore, we combined T-DM1 and volasertib at several different doses and calculated combination indices (CIs) by Bliss Independence Model [28], to check if the two drugs synergistically decrease cell viability. As can be seen in Fig. $2 \mathrm{e}$, in $47 \%$ and $61 \%$ of the doses tested in BT- 474 and SKBR-3 T-DM1R cells, respectively, volasertib synergized with T-DM1 in attenuating cell viability. In order to further support the involvement of PLK1 signaling in T-DM1 resistance, we analyzed a dataset, with mRNA expression of 50 breast cancer cell lines (GSE73526), for which response to a PLK1 inhibitor, GSK461364, is available [29, 30]. Stratification of cell lines with respect to the expression of our T-DM1R signature (see Materials and methods for details) demonstrated that cell lines expected to be more resistant to T-DM1, that is, having high T-DM1R score, had a higher sensitivity to PLK1 inhibition (Fig. 2f). Similar to the proteomic profiling of T-DM1 response of the WT and T-DM1R models (Fig. 1c), we again performed an RPPA experiment: quantifying the same proteins as before, but this time in the resistant cells treated with T-DM1 and volasertib individually or in combination. Strikingly, the profile obtained in the combination therapy perfectly matched with the profile of T-DM1-treated WT cells with respect to the regulation of mitotic arrest and DNA damage markers (Figs. 1c and 2g). These results were further 
A

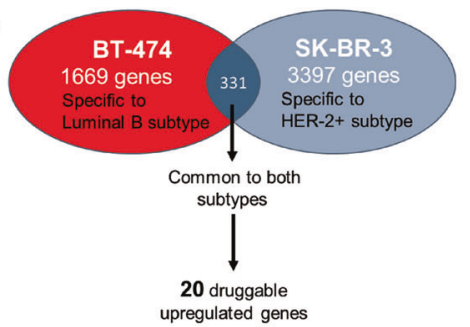

C

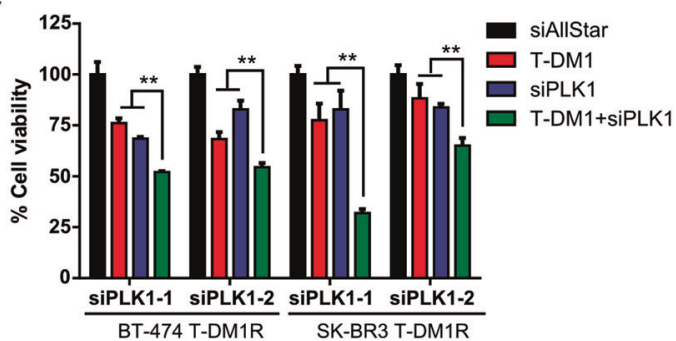

B

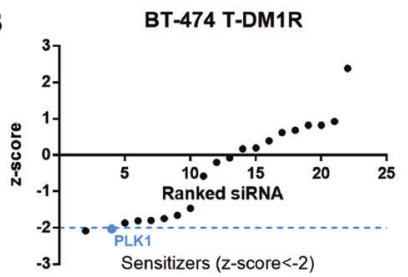

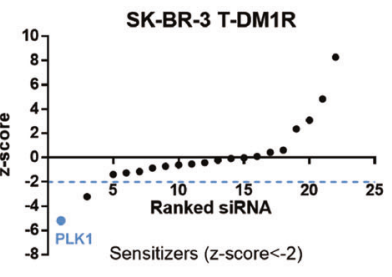

D

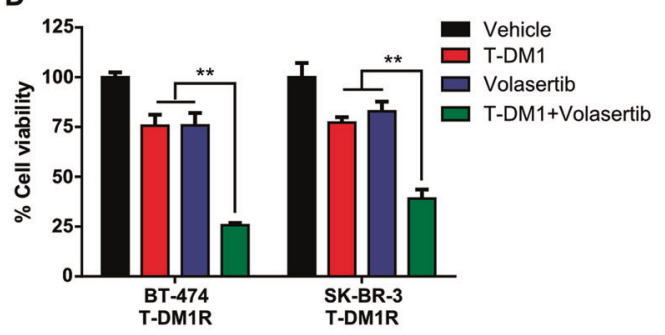

E
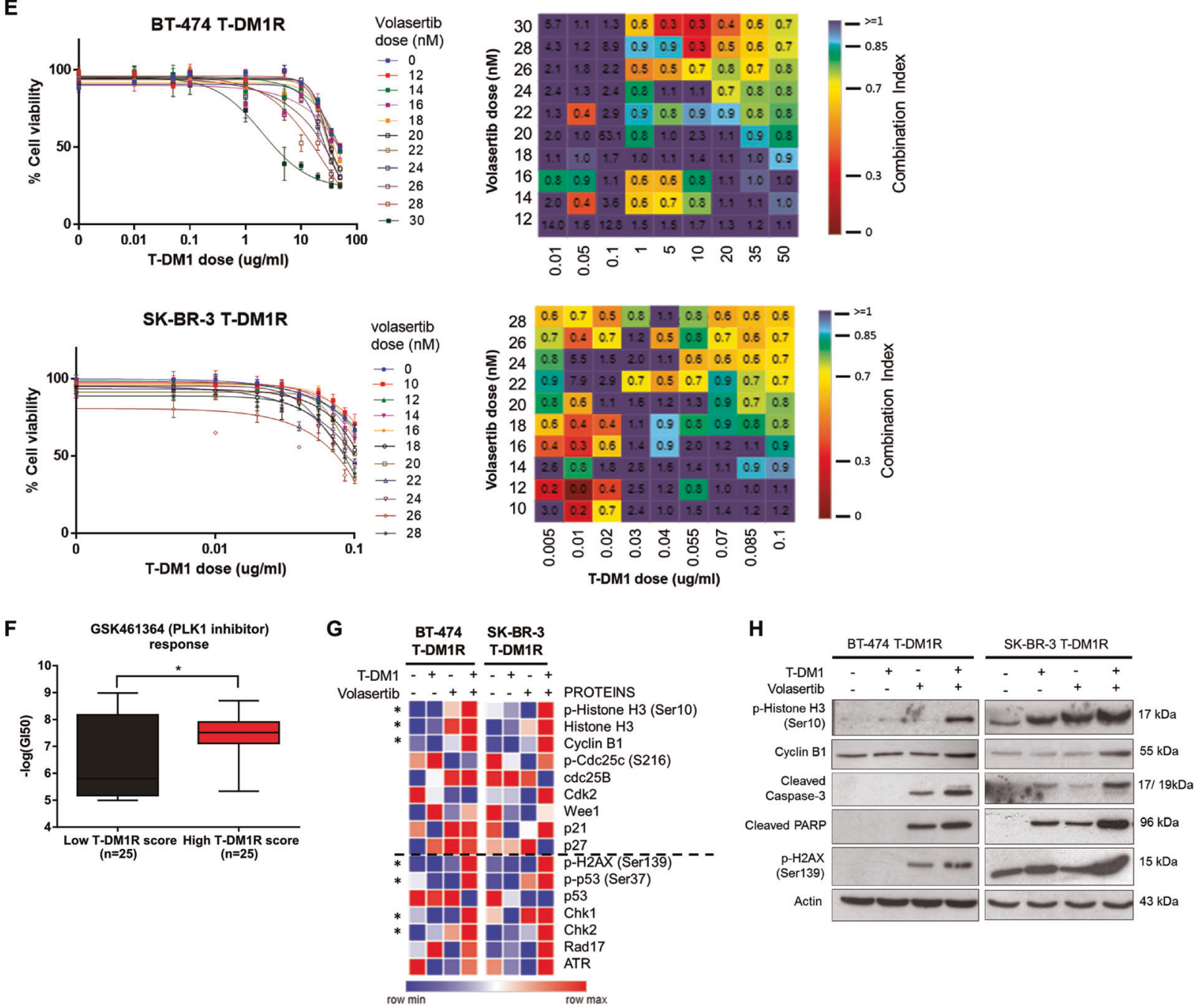
Fig. 2 Whole transcriptome analysis combined with a targeted siRNA sensitization screen identifies PLK1 as a mediator of T-DM1 resistance. a Venn diagram of the significantly differentially expressed genes $(P<0.05)$ between WT and T-DM1R cells for the two models based on the RNA-Seq results. b $Z$-score plots of the siRNA knockdowns performed in the resistant BT-474 (left panel) and SK-BR-3 (right panel) cells in combination with T-DM1. Negative $z$-scores represent knockdowns that cause sensitization to T-DM1 with a $z$ score cutoff of -2 . c Validation of the screen results with two different siRNA sequences against PLK1. Resistant cells were transfected with either non-targeting siAllStar or siPLK1-1 or siPLK1-2 for $24 \mathrm{~h}$, and then treated with 20 and $0.06 \mu \mathrm{g} / \mathrm{ml}$ of T-DM1 for BT-474 and SKBR-3 T-DM1R, respectively, for an additional $72 \mathrm{~h}$. d T-DM1 sensitization with pharmacological PLK1 inhibition by volasertib. BT-474 and SK-BR-3 T-DM1R cells were treated with a combination of TDM1 and volasertib $(20 \mu \mathrm{g} / \mathrm{ml}$ T-DM1 and $25 \mathrm{nM}$ volasertib for BT474 and $0.06 \mu \mathrm{g} / \mathrm{ml} \mathrm{T-DM} 1$ and $15 \mathrm{nM}$ volasertib for SK-BR-3) at the same time for $72 \mathrm{~h}$, and cell viability assay was performed. e Cell viability curves (left panel) and the combination index heatmaps (right panel) for BT-474 T-DM1R and SK-BR-3 T-DM1R cells treated with T-DM1/volasertib combination at indicated doses of the two drugs for $72 \mathrm{~h}$. The synergy score (combination index) was calculated using the Bliss Independence Model. Combination index $<1.0$ indicates synergism. f The response of 50 breast cancer cell lines, separated as TDM1 sensitive and T-DM1 resistant based on the expression of TDM1R gene signature, to a PLK1 inhibitor, GSK461364. The mRNA expression data of the cell lines were obtained from GSE73526, and the $-\log ($ IC50) values for GSK461364 were taken from Daemen et al [30]. g RPPA results for the same set of proteins as in Fig. 1c, this time in T-DM1R cells treated with T-DM1 or volasertib, individually or in combination with doses used in $\mathbf{d}$ for 24 and $48 \mathrm{~h}$ for BT-474 and SKBR-3, respectively. The proteins that showed a consistent change in the two models are highlighted with asteriks. $\mathbf{h}$ Western blot validation of the RPPA data showing the increase in mitotic arrest, apoptosis and DNA damage markers with T-DM1/volasertib combination in TDM1R cells. Box plots depict median number and the 25th to 75th quartiles. Upper and lower whiskers represent the minimum and maximum values in the corresponding group. ${ }^{*} P<0.05$ and $* * P<$ 0.01 . This applies to all figures shown

validated by Western blot analysis of the marker proteins tested in the array also including the apoptotic markers, cleaved caspase-3 and cleaved PARP (Fig. 2h). Altogether, these data suggest a high dependency of T-DM1 resistance on PLK1 overexpression and make PLK1 a highly attractive target in T-DM1 resistance whose inhibition restores all TDM1-associated phenotypes: mitotic arrest, apoptosis and DNA damage.

\section{T-DM1R signature or PLK1 expression correlates with cell cycle and DNA damage and predicts lower sensitivity to taxane/trastuzumab combination in HER2-positive breast cancer patients}

In order to evaluate the clinical relevance of the resistanceassociated phenotypes seen in the in vitro models as well as the relevance of our T-DM1R gene signature, we next mined several breast cancer patient datasets. We started by testing the clinical association of the genes in our T-DM1R signature with the phenotypic processes found to be involved in T-DM1 resistance in breast cancer, in general. For this purpose, we analyzed The Cancer Genome Atlas breast cancer data (http://cancergenome.nih.gov), which contains high number of patients with scores available for different cancer-related processes, including cell cycle and DNA damage response scores. After assigning a T-DM1R signature score to each patient, we correlated this score with the available scores of cancer-related processes [31] (see Materials and methods for details). Similar to the phenotypes that we had seen in vitro, T-DM1R signature positively correlated with an active cell cycle and DNA damage response in patients as well (Fig. 3a). Since gene expression datasets of T-DM1-treated patients are not yet available, we instead analyzed a dataset of taxane-treated breast cancer patients where HER2-positive ones also received trastuzumab (GSE42822). This type of combination therapy, that is, trastuzumab and the microtubule-targeting taxanes, mimics treatment with T-DM1, which is a conjugate of trastuzumab and DM1. Among the combination-treated HER2-positive patients, T-DM1R signature score was found to be higher when there is residual disease, as compared to complete pathological response. On the other hand, such a difference in the resistance score was not observed among HER2negative patients treated only with taxane-containing chemotherapy (Fig. 3b). Finally, we performed a gene set enrichment analysis (GSEA) in a similar patient dataset (GSE22358) where 32 of the HER2-positive breast cancer patients received trastuzumab and docetaxel/capecitabine combination, which again mimicks T-DM1 treatment [32]. Patients who received this combination and at the same time expressed higher PLK1 had a significant enrichment of mitotic progression and DNA repair gene sets, which further suggested PLK1 overexpression as a positive regulator of mitotic progression and DNA repair, specifically in $\mathrm{T}$ DM1 resistance (Fig. 3c). Altogether, these data identified PLK1 as a clinically relevant druggable target in T-DM1 resistance by favoring evasion from drug-induced mitotic arrest, apoptosis and DNA damage both in vitro and in patients.

\section{PLK1-mediated T-DM1 resistance involves rewiring of apoptotic signaling, and is mainly mediated by Bcl-2 and its partner Bim}

Similar to a dependency on caspase activity for T-DM1 cytotoxicity in WT cells (Fig. 1i), T-DM1 sensitization with volasertib was also dependent on caspase activation (Fig. 4a). This prompted us to screen a panel of pro-apoptotic and anti-apoptotic markers for differential regulation in combination-treated resistant cells which may provide a clue on how PLK1 inhibition in T-DM1R models enhances apoptotic cell death (Fig. 4b). Among the screened proapoptotic and anti-apoptotic proteins, Bcl-2 
A

Correlation of T-DM1R Signature with Cellular Processes

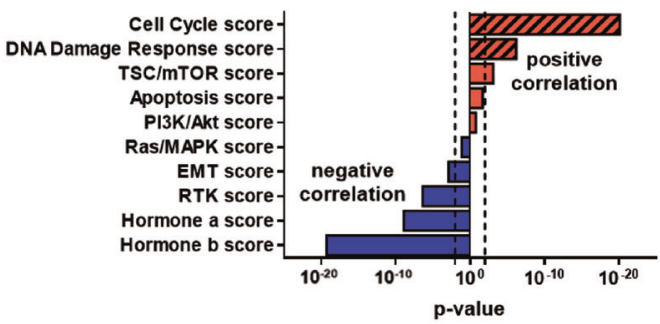

B
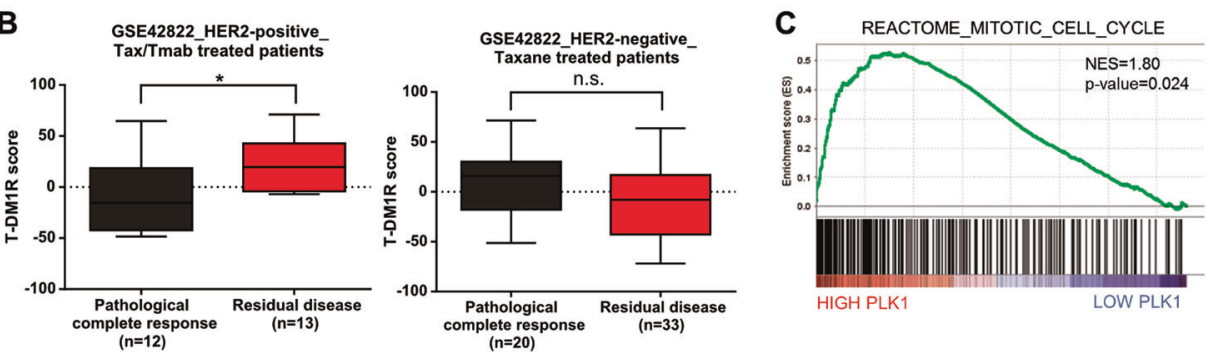
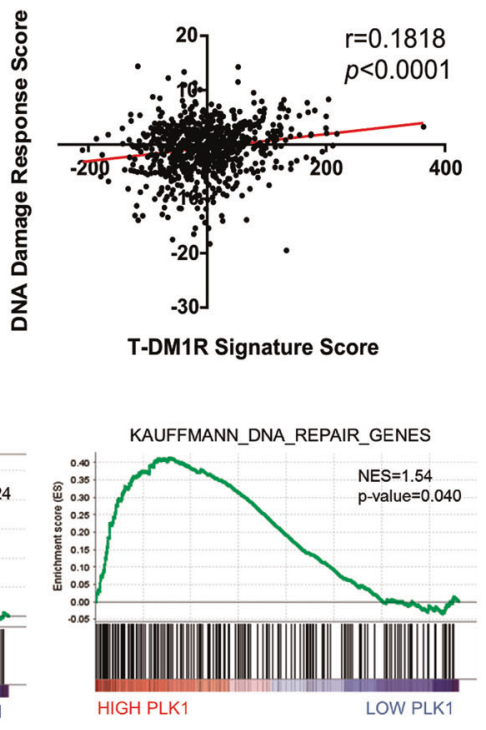

Fig. 3 T-DM1R signature or PLK1 expression correlates with cell cycle and DNA damage, and predicts lower sensitivity to taxane/ trastuzumab combination in HER2-positive breast cancer patients. a Correlation between the T-DM1R signature and the cancer-associated process scores of TCGA breast cancer patients validating the clinical relevance of the resistance-associated processes identified in vitro. Correlation of T-DM1R signature with cell cycle and DNA damage scores were given as separate XY plots. b T-DM1R signature score in

phosphorylation at serine 70 (Ser70) was enhanced upon combination of T-DM1 with volasertib (Fig. 4b). Increase in phosphorylation at this site was also evident in T-DM1treated WT cells (Fig. 4c). This phosphorylation site was previously associated with prolonged mitosis $[33,34]$ and has shown to inhibit the anti-apoptotic activity of Bcl-2 [34-36]. Therefore, we reasoned that the volasertib-induced phosphorylation of Bcl-2 in T-DM1-treated resistant cells would inhibit the sequestration of the pro-apoptotic proteins by Bcl-2, and thereby initiate apoptosis. Indeed, coimmunoprecipitation with one of the pro-apoptotic proteins, Bim, demonstrated a loss of Bcl-2 binding to Bim upon combination treatment in both resistant models (Fig. 4d) correlating with the increase in Ser70 phosphorylation of Bcl-2 (Fig. 4b). Displacement of Bim from Bcl-2 was also detected in T-DM1-treated WT cells which was nearly absent in the resistant models (Fig. 4e). However, the interaction of Bim with Mcl-1, which is another antiapoptotic protein, remained unchanged, and therefore suggests a Bcl-2-specific regulation in T-DM1 resistance (Fig. $4 \mathrm{~d}$, e). Overall, these results demonstrate that Ser70 phosphorylation of Bcl-2 upon T-DM1/volasertib treatment acts as an inhibitory regulation which weakens the interaction between Bcl-2 and the pro-apoptotic activator, Bim, in order to prevent its sequestration by Bcl-2. This ultimately leads to apoptotic cell death in the resistant models, similar to the cytotoxic effects of T-DM1 on WT cells.
HER2-positive (left) and HER2-negative (right) breast cancer patients (GSE42822) grouped according to their clinical response to taxane/ trastuzumab combination or only taxane treatment for HER2-positive and HER2-negative patients, respectively. $\mathbf{c}$ GSEA analysis of mitosis and DNA repair-related gene sets in taxane/trastuzumab combinationtreated HER2-positive breast cancer patients (GSE22358) with respect to their PLK1 expression. $* P<0.05, N S$ not significant

Strikingly, the basal expression of $\mathrm{Bcl}-2$ was strongly upregulated in both T-DM1R models at both mRNA and protein levels (Fig. 4f). Moreover, the resistant models were found to be more sensitive to a $\mathrm{BH} 3$ mimetics, ABT-263, which inhibits the interaction between the Bcl-2 family proteins, and thereby prevents the sequestration of the proapoptotics by the anti-apoptotic members [37] (Supplementary Figure S4). The increased dependency on Bcl-2 had indeed functional consequences in T-DM1 resistance as combining T-DM1 with ABT-263 led to the strongest growth inhibition as compared to individual treatments although to a lesser extent than PLK1 inhibition (Fig. 4g). Furthermore, the two drugs were combined at a wide range of doses of the two inhibitors, and a synergistic growth inhibition was observed at $64 \%$ and $80 \%$ of the doses tested for BT-474 and SK-BR-3 T-DM1R cells, respectively (Fig. 4h). The synergistic effect of T-DM1 and ABT-263 on inhibiting cell viability was mainly through induction of apoptotic cell death as evident by the drastic increase in the levels of cleaved caspase- 3 and cleaved PARP upon combination treatment with almost no change in the expression of mitotic arrest markers (Fig. 4i, Supplementary Figure S5). Overall, these data indicate that PLK1-mediated TDM1 resistance involves rewiring of apoptotic signaling, mainly mediated by Bcl-2 and its partner Bim, and targeting Bcl-2 synergizes with T-DM1 to reduce viability of T-DM1 resistant cells. 
A

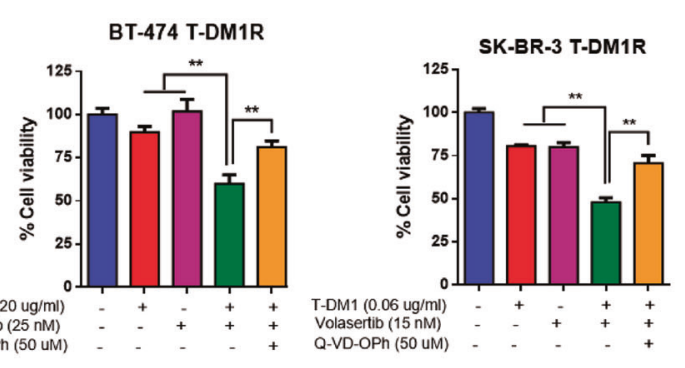

C

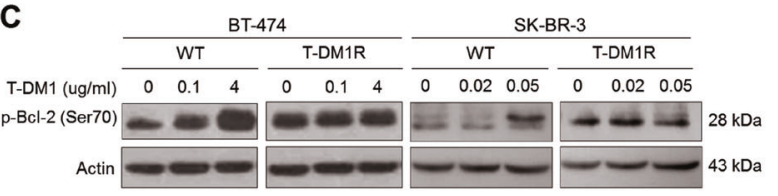

E

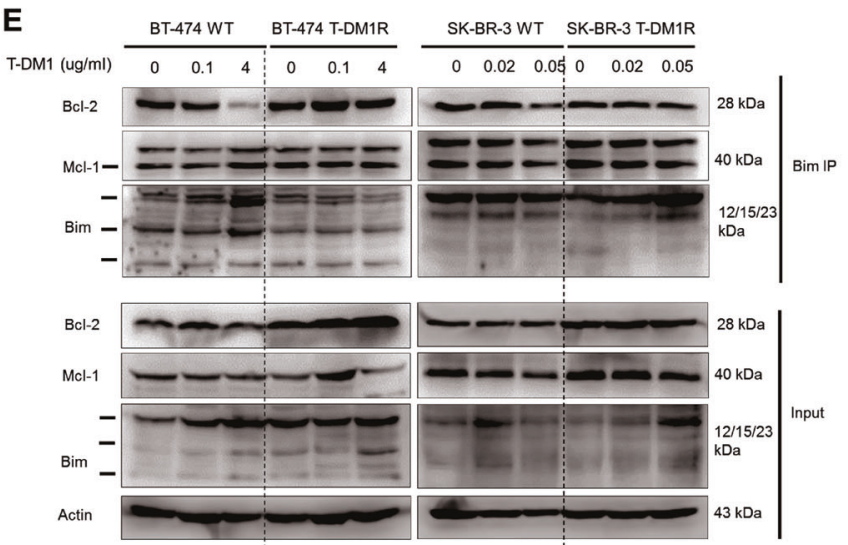

F

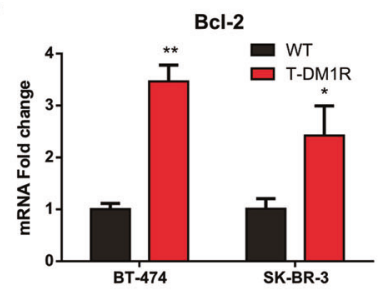

H
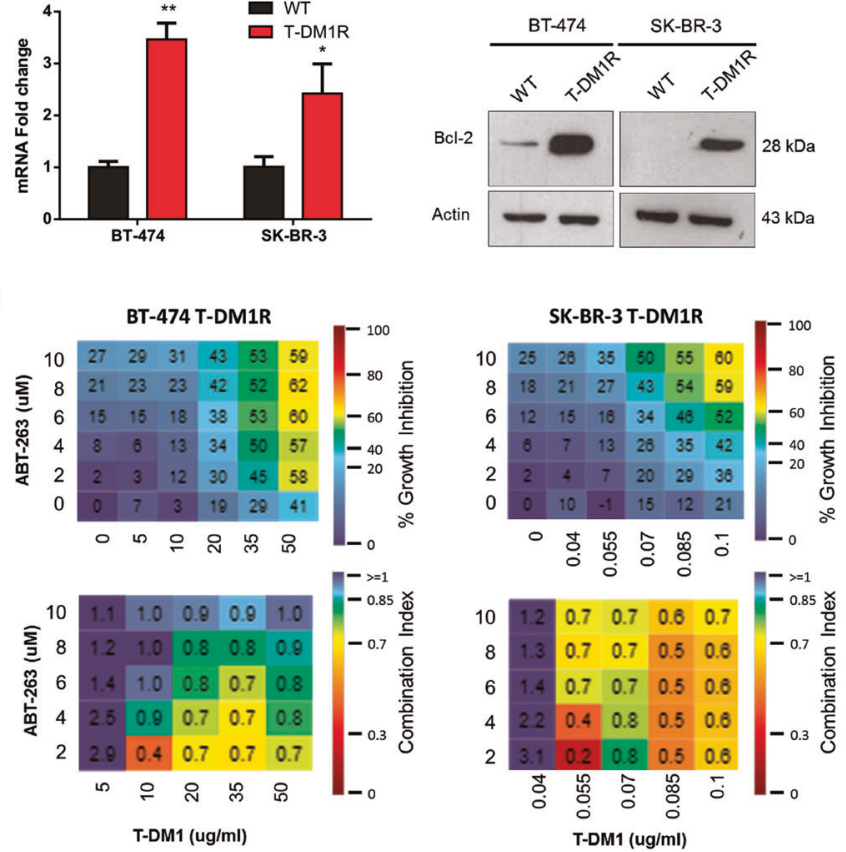

B

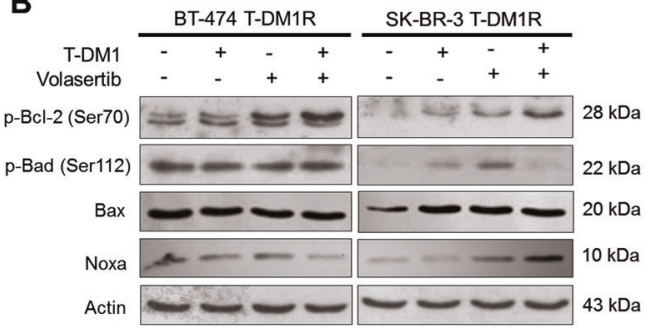

D

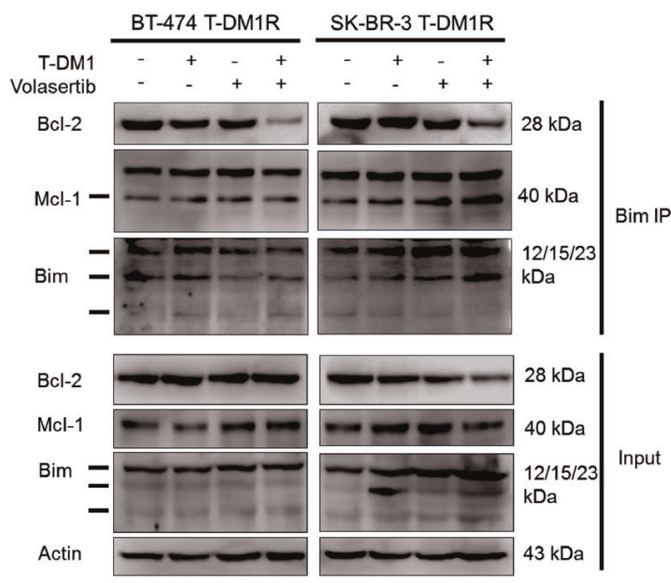

G
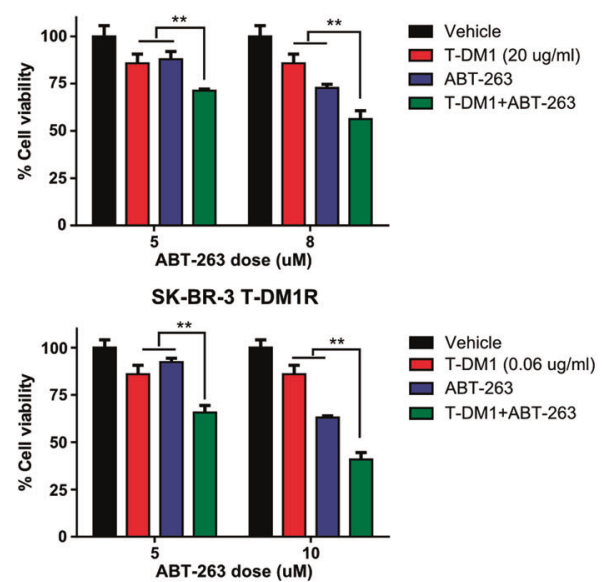

I

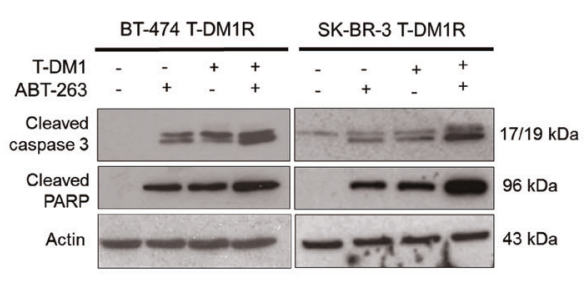


Fig. 4 PLK1-mediated T-DM1 resistance involves rewiring of apoptotic signaling and is mainly mediated by Bcl-2 and its partner Bim. a Loss of cell growth inhibition in T-DM1/volasertib combinationtreated BT-474 and SK-BR-3 T-DM1R cells upon inhibition of caspases by Q-VD-OPh. Cells were pre-treated with $50 \mu \mathrm{M}$ of Q-VD-OPh for $1 \mathrm{~h}$ preceding T-DM1 and volasertib treatments for $72 \mathrm{~h}$. Twenty and $0.06 \mu \mathrm{g} / \mathrm{ml}$ of T-DM1 and 25 and $15 \mathrm{nM}$ of volasertib was used for BT-474 and SK-BR-3, respectively. b Western blot analysis of the apoptosis-related proteins in resistant models treated with individual or combination therapies of T-DM1 and volasertib at the doses used in a for $24 \mathrm{~h}$. Actin was used as a loading control. $\mathbf{c}$ Bcl-2 phosphorylation at Ser70 residue in BT-474 and SK-BR-3 WT cells treated with 4 and $0.05 \mu \mathrm{g} / \mathrm{ml}$ of T-DM1, respectively. d Co-immunoprecipitation for the interaction of Bcl-2 and Mcl-1 with the pro-apoptotic Bim in resistant cells treated with T-DM1/volasertib combination at the doses used in a for $24 \mathrm{~h}$. Inputs for co-immunoprecipitations were also subjected to immunoblot analysis (lower panel) for the same proteins. e Coimmunoprecipitation analysis of the same set of proteins as in $\mathbf{d}$, in WT cells treated with 4 and $0.05 \mu \mathrm{g} / \mathrm{ml}$ of T-Dm1 for $24 \mathrm{~h}$ for BT- 474 and SK-BR-3, respectively. f qRT-PCR and Western blot analysis of Bcl-2 in WT versus T-DM1R models showing overexpression in resistance. $\mathbf{g}$ T-DM1 sensitization of the resistant models with the Bcl2 family inhibitor, ABT-263. Cells were treated with 20 and $0.06 \mu \mathrm{g} /$ $\mathrm{ml}$ of T-DM1 and/or 8 and $10 \mu \mathrm{M}$ of ABT-263, for BT-474 and SKBR-3, respectively, at the same time for $72 \mathrm{~h}$. h Heatmaps of \% growth inhibition (upper panel) and combination index (lower panel) in BT474 and SK-BR-3 T-DM1R cells treated with T-DM1/ABT-263 combination at different doses of the two drugs for $72 \mathrm{~h}$. The synergy score (combination index) was calculated using the Bliss Independence Model. Combination index $<1.0$ indicates synergism. i Western blot analysis of the apoptotic markers in T-DM1R cells treated with 20 and $0.06 \mu \mathrm{g} / \mathrm{ml}$ of T-DM1 in combination with 8 and $10 \mu \mathrm{M}$ of ABT263 for BT-474 and SK-BR-3 T-DM1R, respectively. A strong synergy between T-DM1 and ABT-263 in inducing apoptotic cell death was observed. $* * P<0.01$

\section{T-DM1/volasertib combination leads to apoptosis and DNA damage in a SAC-dependent manner through CDK1-dependent BCl-2 phosphorylation}

PLK1 inhibition often leads to large numbers of spindle defects and formation of monopolar spindles resulting in spindle checkpoint activation and subsequent prolonged mitosis [38, 39]. In order to see if the mitotic arrest induced by T-DM1/volasertib combination in the T-DM1R models is also attributed to spindle checkpoint activation, we inhibited SAC with an inhibitor of the spindle checkpoint kinase, Mps1 (TC Mps1). Treating cells with TC Mps1, after $24 \mathrm{~h}$ of combination treatment (T-DM1 and volasertib) markedly decreased the accumulation of cyclin B1 and the level of phosphorylated histone $\mathrm{H} 3$, indicating mitotic slippage upon blockage of SAC (Fig. 5a). A similar SAC dependency for mitotic arrest was also present in WT cells under T-DM1 treatment, suggesting that the T-DM1resistant models found a way to override SAC in a PLK1dependent manner (Fig. 5b). In order to further demonstrate the importance of PLK1 overexpression in evasion from the SAC-dependent mitotic arrest, we overexpressed PLK1 in
WT cells, in the presence of T-DM1, and analyzed the changes in the expression of mitotic arrest and apoptosis markers. As can be seen in Supplementary Figure S6, introducing PLK1 to WT cells under T-DM1 treatment caused loss of mitotic arrest and apoptosis that was substantially rescued upon inhibition of PLK1 with volasertib. These results suggest that PLK1 overexpression could override SAC-induced mitotic arrest and subsequent apoptosis in T-DM1 resistance.

It is noteworthy that the loss of mitotic arrest upon SAC inhibition also impaired the DNA damage response elicited after treatment with T-DM1/volasertib combination in the resistant models as well as in sensitive cells under T-DM1 treatment, thus linking DNA damage to prolonged mitosis (Fig. 5a, b). The activation of caspase-activated DNase enzyme is one of the proposed mechanisms through which cells may gain chromosomal breaks upon prolonged mitosis $[40,41]$. In order to elucidate if the DNA damage response in our system also displays a similar regulation that is dependent on caspase activity, we inhibited caspases with $\mathrm{Q}-\mathrm{VD}-\mathrm{OPh}$ and observed a decrease in the formation of $\mathrm{p}$ H2AX foci upon caspase inhibition (Fig. 5c, d and Supplementary Figure S7). Altogether, our results suggest a sequence of events that is initiated by a SAC-dependent mitotic arrest, followed by caspase activation and apoptosis which also leads to DNA breaks and formation of DNA damage response upon T-DM1 or combination treatment in WT and T-DM1R models, respectively.

Next, we sought to uncover how Bcl-2 is phosphorylated during the events starting from SAC activation and continuing towards apoptotic cell death. We saw that Bcl-2 phosphorylation was reduced strongly with mitotic slippage after TC Mps1 treatment (Fig. 5e, f). This observation suggests a mitotic arrest-dependent phosphorylation of Bcl2. Accumulation of cyclin B1/CDK1 complex in mitotically arrested cells makes CDK1 an attractive kinase candidate that can mediate Bcl-2 phosphorylation. To test this, we inhibited CDK1 using a CDK1-specific inhibitor, RO-3306, in combination-treated resistant cells or T-DM1-treated WT cells. CDK1 inhibition resulted in loss of Bcl-2 phosphorylation accompanied by a decrease in the apoptotic marker, cleaved PARP, although cells displayed similar levels of mitotic arrest (Fig. 5g, h). This clearly indicates that the CDK1-dependent Bcl-2-phosphorylation is required for apoptotic cell death in prolonged mitosis.

\section{PLK1 inhibition overcomes de novo T-DM1 resistance as well via phosphorylation of $\mathrm{BCl}-\mathrm{xL}$ in a CDK1-dependent manner}

In order to extend the sensitizer role of PLK1 to de novo TDM1 resistance, we used the HER2-positive, de novo trastuzumab-resistant cell line JIMT-1 [42], which we 
A

\begin{tabular}{rlllllllllll} 
& \multicolumn{3}{c}{ BT-474 T-DM1R } & & \multicolumn{3}{c}{ SK-BR-3 T-DM1R } \\
\cline { 2 - 8 } T-DM1 & - & + & - & + & + & & - & + & - & + & + \\
Volasertib & - & - & + & + & + & & - & - & + & + & + \\
TC Mps1 & - & - & - & - & + & - & - & - & - & +
\end{tabular}

p-Histone H3 (Ser10)

Cyclin B1

p-H2AX (Ser139)

Actin

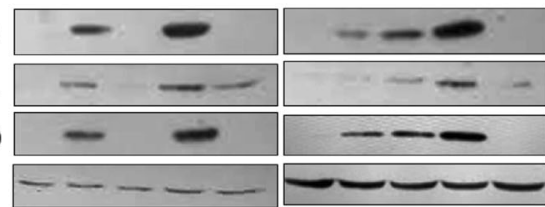

$17 \mathrm{kDa}$

$55 \mathrm{kDa}$

$15 \mathrm{kDa}$

$43 \mathrm{kDa}$

C

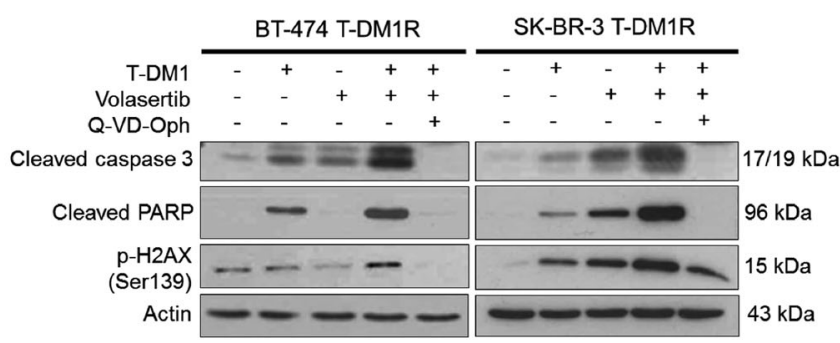

E

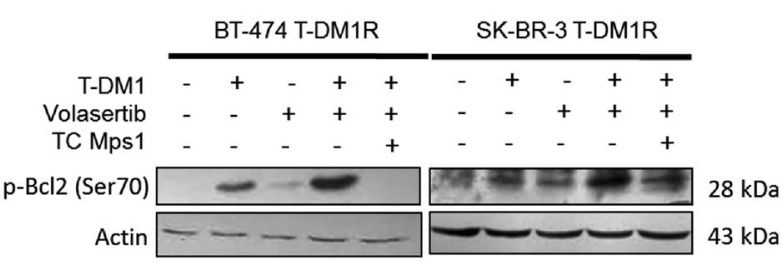

G

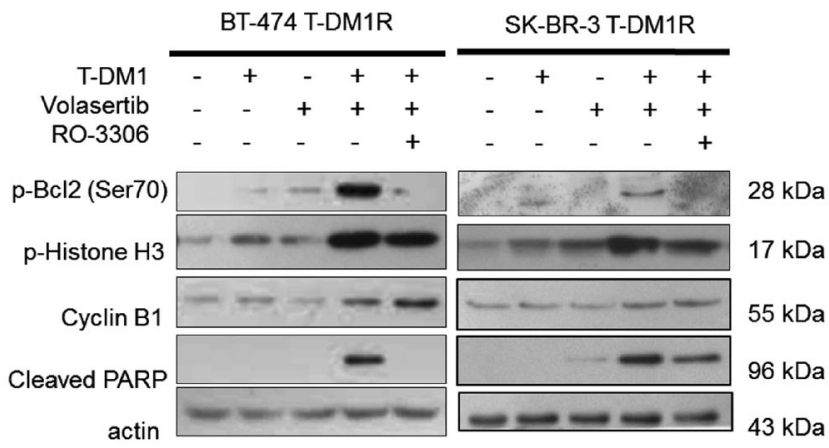

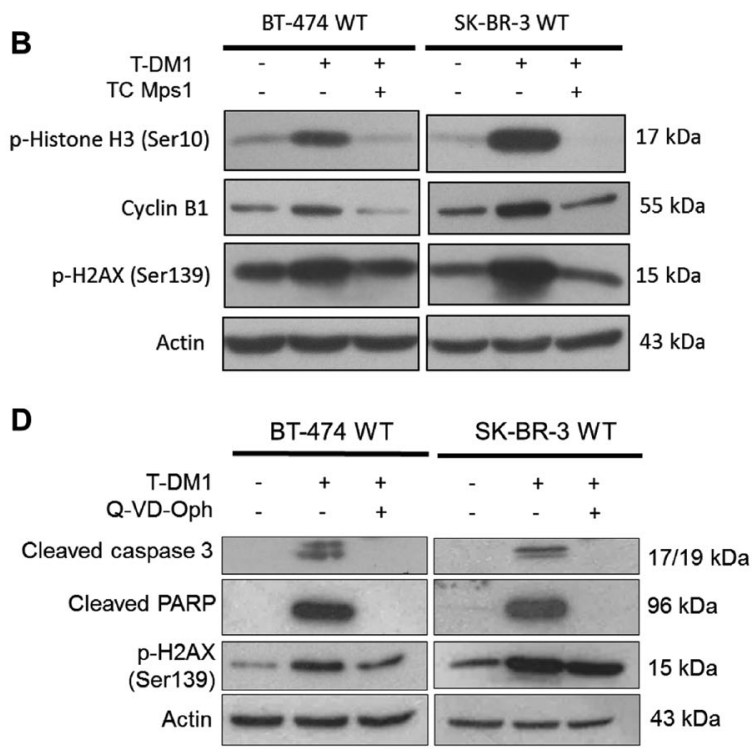

$\mathbf{F}$

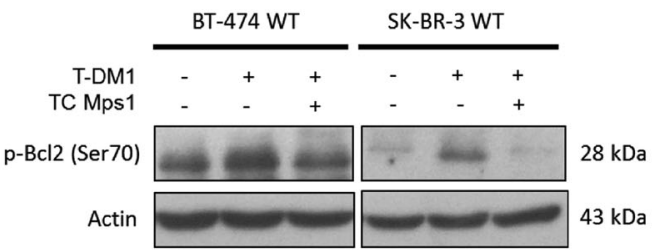

H

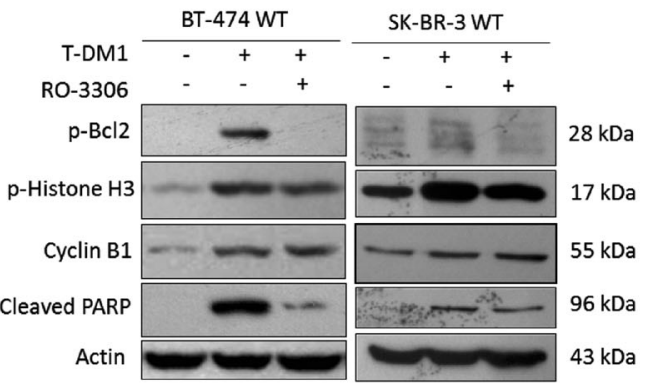

Fig. 5 T-DM1/volasertib combination leads to apoptosis and DNA damage in a spindle checkpoint-dependent manner through CDK1dependent Bcl-2 phosphorylation in mitotically arrested cells. a, b Western blot analysis of cyclin B1 and p-Histone H3 (Ser10) showing mitotic slippage in T-DM1/volasertib-treated (a) and T-DM1-treated (b) T-DM1R and WT cells, respectively, upon $6 \mathrm{~h}$ treatment with a spindle checkpoint kinase (Mps1) inhibitor, TC Mps1. T-DM1R cells were treated with 20 and $0.06 \mu \mathrm{g} / \mathrm{ml} \mathrm{T-DM1}$, in combination with 25 and $15 \mathrm{nM}$ volasertib for BT-474 and SK-BR-3 T-DM1R, respectively, for $24 \mathrm{~h}$, followed by $6 \mathrm{~h}$ treatment with $1 \mu \mathrm{M}$ TC Mps1 (A). WT cells were treated with 4 and $0.05 \mu \mathrm{g} / \mathrm{ml}$ of T-DM1 for BT-474 and SK-BR-3 WT, respectively, for $24 \mathrm{~h}$, followed by $6 \mathrm{~h}$ treatment with $1 \mu \mathrm{M}$ TC Mps1 (b). c, d Western blot analysis of DNA damage marker, p-H2AX (Ser139), in resistant (c) and WT (d) cells pre-treated with $50 \mu \mathrm{m}$ of the pan-caspase inhibitor, Q-VD-OPh, for $1 \mathrm{~h}$, followed

identified as being de novo resistant also to T-DM1 (Fig. 6a). First, we tested if the de novo resistant JIMT-1 cells may also be responsive to volasertib and ABT-263 by $24 \mathrm{~h}$ of combination or T-DM1 treatments, respectively, at the doses used in a and b. Loss of p-H2AX (Ser139) upon caspase inhibition indicates caspase-dependent DNA damage. e, f Western blot analysis of p-Bcl-2 (Ser70) in resistant (e) and WT (f) cells from a and b, showing a mitotic phosphorylation of Bcl-2. $\mathbf{g}$, h Western blot analysis of p-Bcl-2, apoptotic and mitotic markers, showing a Bcl-2 phosphorylation-dependent apoptosis via CDK1, in combinationtreated resistant (g) and T-DM1-treated WT cells (h). T-DM1R cells were treated with combination therapy at the doses used in a for 24 and $30 \mathrm{~h}$, followed by 2 and $1 \mu \mathrm{M}$ of the CDK1 inhibitor, RO-3306, for an additional 12 and $18 \mathrm{~h}$ for BT-474 and SK-BR-3, respectively (g). WT cells were treated with T-DM1 at the doses used in b for 24 and $36 \mathrm{~h}$, followed by 2 and $1 \mu \mathrm{M}$ of the CDK1 inhibitor, RO-3306, for an additional 12 and $6 \mathrm{~h}$ for BT-474 and SK-BR-3, respectively (h)

combination under T-DM1 treatment, similar to acquired resistant models. Indeed, we observed high level of sensitization to T-DM1 upon combination of T-DM1 with either 
A

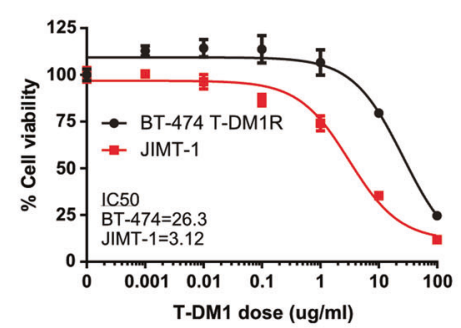

D

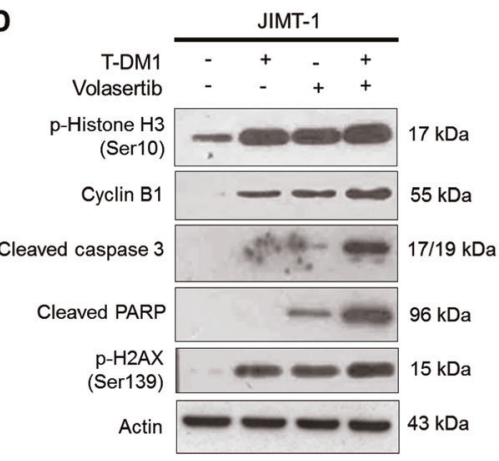

B
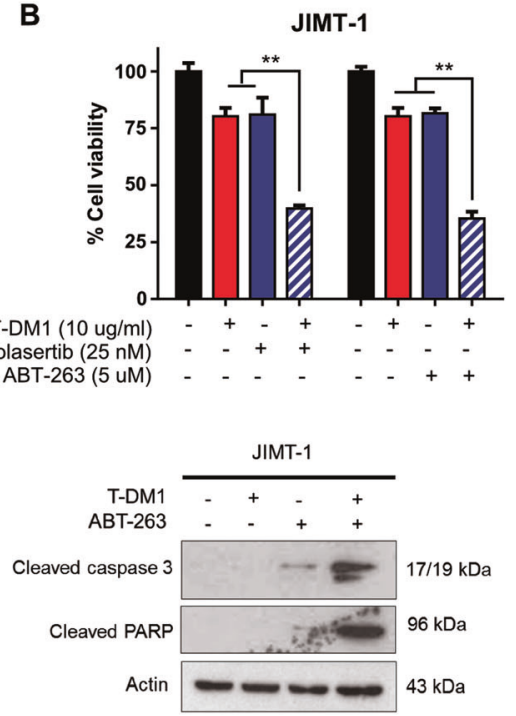

C
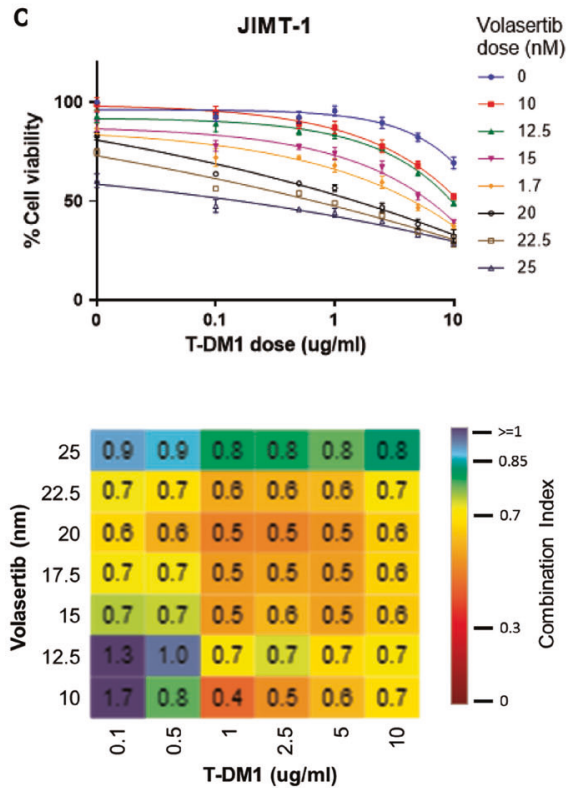

E

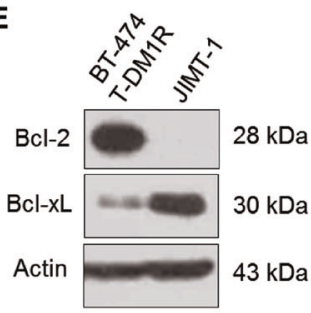

$\mathbf{F}$

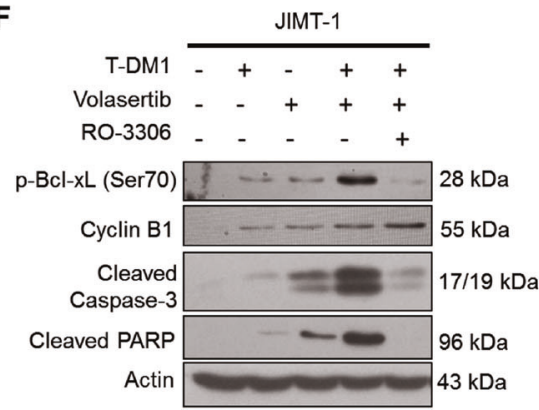

Fig. 6 PLK1 inhibition overcomes de novo T-DM1 resistance as well via phosphorylation of $\mathrm{Bcl}-\mathrm{xL}$ in a CDK1-dependent manner. a Dose-response curves of BT-474 T-DM1R and JIMT-1 cells treated with increasing doses of T-DM1 for $72 \mathrm{~h}$. b T-DM1 sensitization of JIMT-1 cells with volasertib or ABT-263. Cells were treated with 10 $\mu \mathrm{g} / \mathrm{ml}$ of T-DM1 in combination with either $25 \mathrm{nM}$ of volasertib or 5 $\mu \mathrm{M}$ of ABT-263 at the same time for $72 \mathrm{~h}$. c Growth inhibition curves (above) and the combination index heatmap (below) for JIMT-1 cells treated with T-DM1/volasertib combination at indicated doses of the two drugs for $72 \mathrm{~h}$. The synergy score (combination index) was calculated using the Bliss Independence Model. Combination index $<1.0$ indicates synergism. d Western blot analysis of the markers for mitotic arrest, apoptosis and DNA damage in JIMT-1 cells under $24 \mathrm{~h}$ of TDM1/volasertib (left panel) or $48 \mathrm{~h}$ of T-DM1/ABT-263 (right panel) treatments at doses used in $\mathbf{b}$. e Expression of Bcl-2 and Bcl-xL in the acquired resistant BT-474 models and the de novo resistant JIMT-1 cell line showing a strong overexpression of $\mathrm{Bcl}-\mathrm{xL}$ in de novo resistance. $\mathbf{f}$ Western blot analysis of Bcl-xL in JIMT-1 cells treated with combination treatment for $18 \mathrm{~h}$ at doses used in $\mathbf{b}$, followed by 18 $\mathrm{h}$ of $2 \mu \mathrm{M}$ RO-3306 treatment, showing loss of Ser62 phosphorylation upon CDK1 inhibition. $* * P<0.01$ volasertib or ABT-263 (Fig. 6b). Synergism between TDM1 and volasertib was verified by combining the two drugs at a wide range of doses (Fig. 6c). Most importantly, sensitization was again accompanied by increases in markers of mitotic arrest, apoptosis and DNA damage (Fig. 6d). Next, we wanted to test if volasertib-mediated apoptosis is also dependent on CDK1-mediated Bcl-2 phosphorylation in de novo resistance. First, we examined if $\mathrm{Bcl}-2$ is expressed at high levels in JIMT-1 cells as it is in acquired resistant models. However, we saw almost no expression of the $\mathrm{Bcl}-2$ protein in these cells, suggesting that this is mediated via regulation of an anti-apoptotic protein other than $\mathrm{Bcl}-2$ that, nevertheless, is also targeted by ABT-263. Anti-apoptotic Bcl-xL has also been shown to be targeted by ABT-263 [37]. Indeed, Western blot analysis of Bcl-xL revealed a very high expression of Bcl-xL in JIMT-1 cells as compared to the BT-474 T-DM1R model (Fig. 6e). Interestingly, we detected an increased phosphorylation of Bcl-xL at position Ser62, which is homologous to Ser70 of Bcl-2, upon combination treatment (Fig. 6f). Furthermore, 

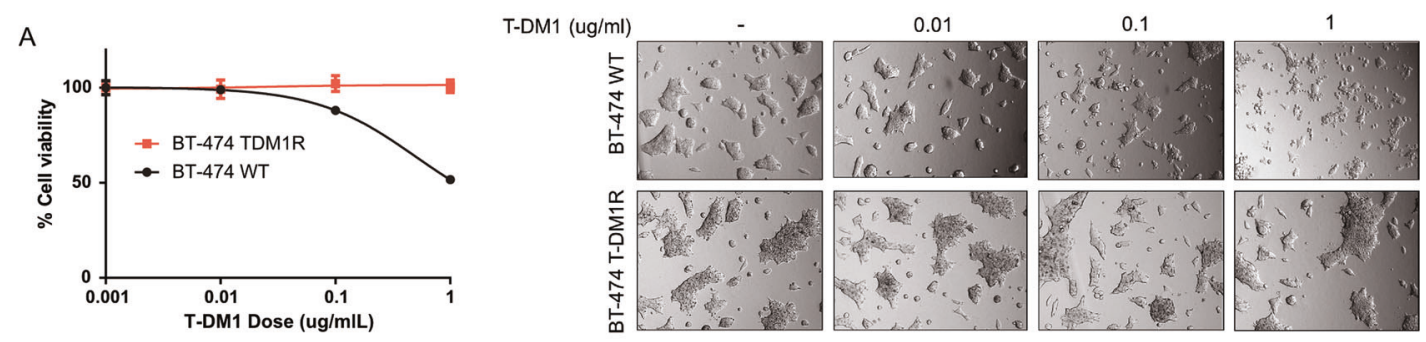

B
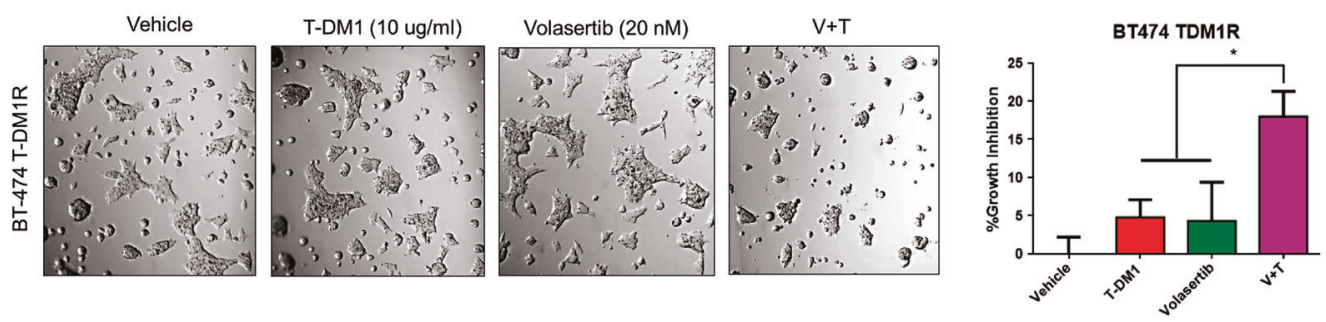

C
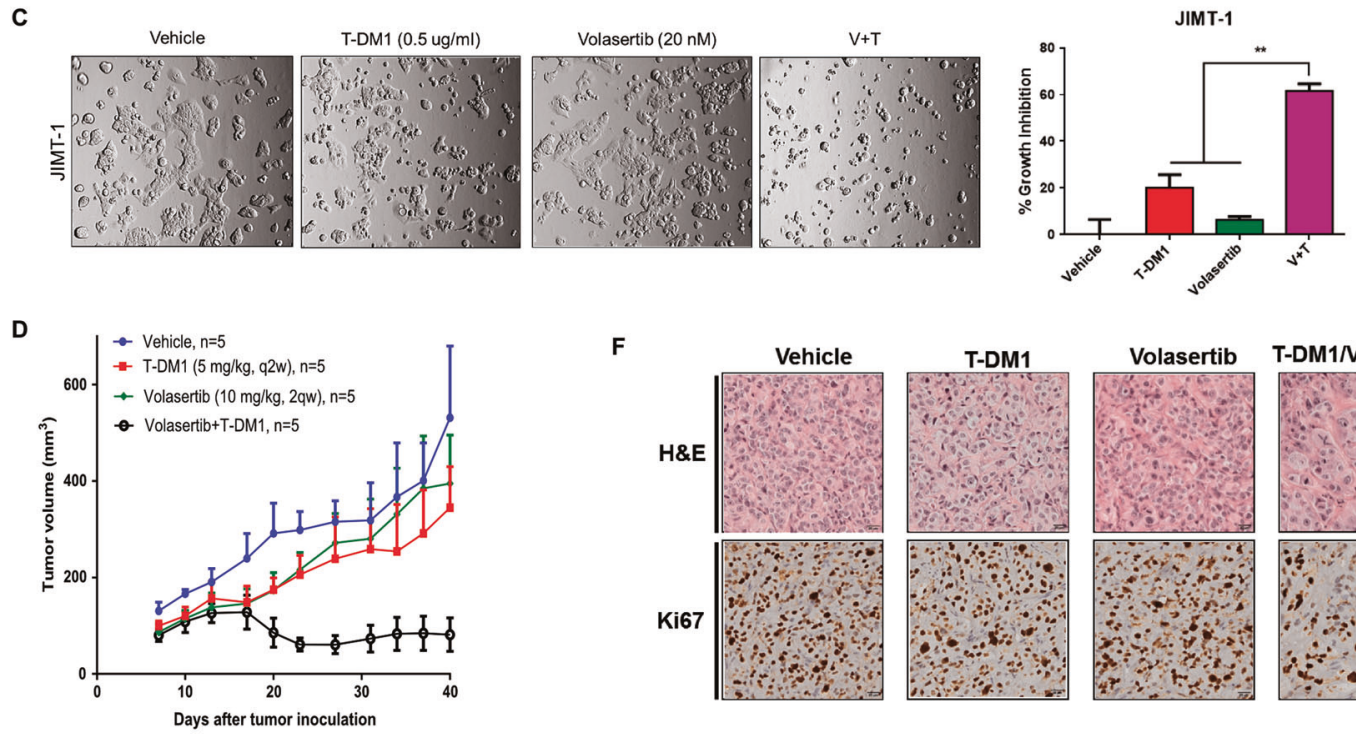

$\mathbf{F}$
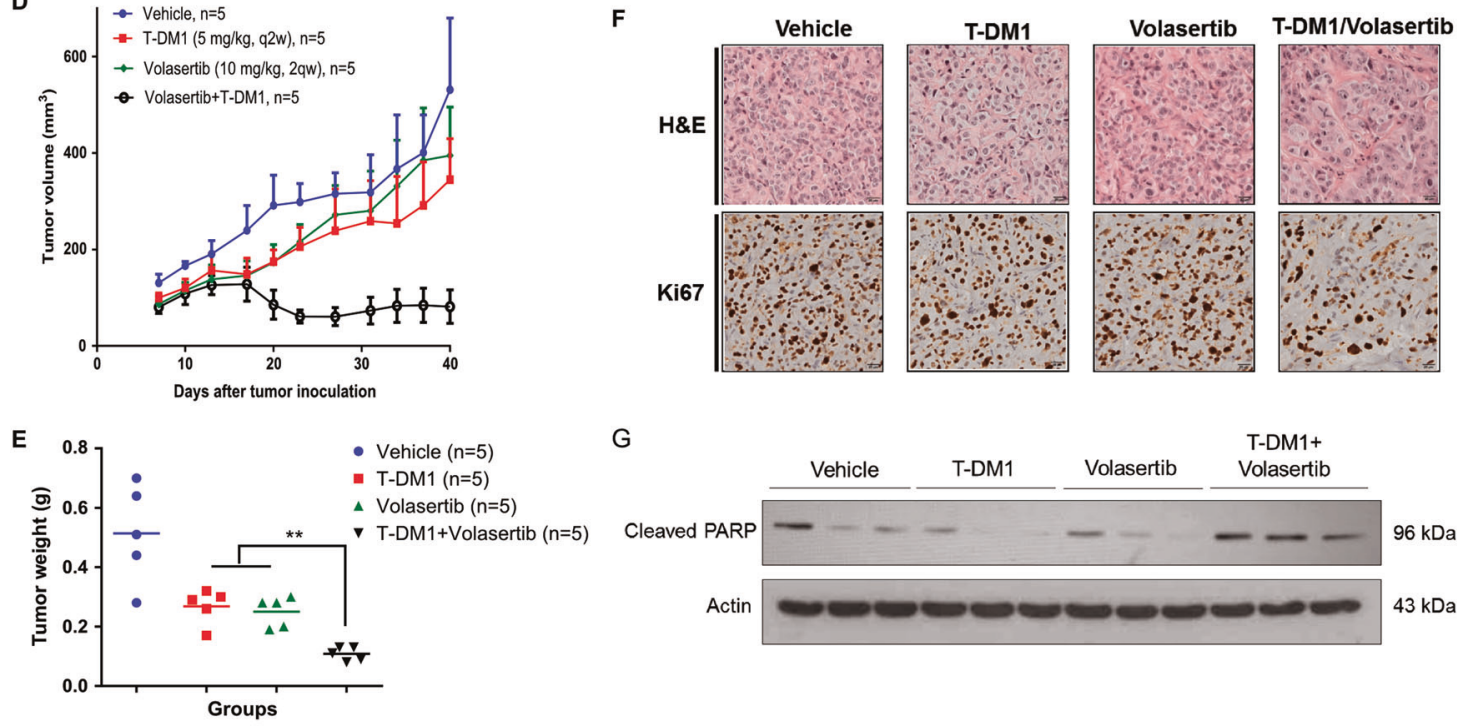

H

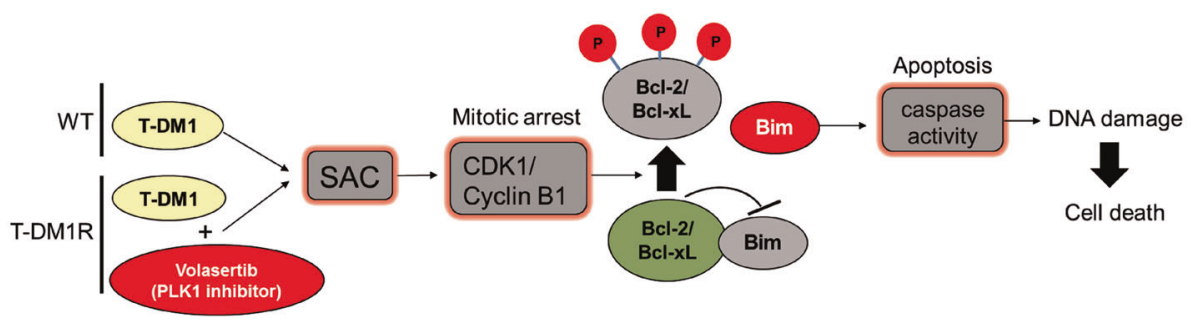

inhibition of CDK1 activity with RO-3306 caused Bcl-xL dephosphorylation and subsequent decrease in apoptosis without inducing mitotic slippage, similar to what we had observed at the Ser70 site of Bcl-2 in acquired resistant models (Figs. 6f and $5 \mathrm{~g}, \mathrm{~h}$ ). These data provided further support for a more generalized regulation of the anti- 
Fig. 7 Volasertib overcomes T-DM1 resistance in 3D culture and in vivo xenografts. a Differential response of BT-474 WT and TDM1R cells to T-DM1 in 3D culture. Cells were seeded on Matrigelcoated 96-well plate with a density of 10,000 cells per well. T-DM1 treatment was done at the indicated doses at the same time of cell seeding, and cell growth was measured by a CellTiter-Glo assay after $72 \mathrm{~h}$. Representative images are provided on the left panel, and \% cell viability is given on the right panel. b, c T-DM1 sensitization with volasertib in 3D models of BT-474 T-DM1R (b) and JIMT-1 (c) cells. Cells were seeded on Matrigel-coated 96-well plate with a cell density of 10,000 and 6000 cells per well, and treated with 10 and $0.5 \mu \mathrm{g} / \mathrm{ml} \mathrm{T}$ DM1 in combination with $20 \mathrm{nM}$ volasertib for BT-474 T-DM1R and JIMT-1, respectively. The results in $\mathbf{a}-\mathbf{c}$ are shown as average of three technical replicates. d Tumor growth in JIMT-1 xenografts treated with T-DM1 and volasertib, individually or in combination. Treatments were started at day 10 after tumor inoculation when tumor volumes reached $100-200 \mathrm{~mm}^{3}$. Mice were treated with $5 \mathrm{mg} / \mathrm{kg} \mathrm{T}$ DM1 (once in 2 weeks intravenously), volasertib (twice a week orally) or combination of the two for 30 days. e Tumor weights from mice treated with vehicle, T-DM1, volasertib or the combination from $\mathbf{d} . \mathbf{f}$ $\mathrm{H} \& \mathrm{E}$ and Ki67 staining of the tumors from d. $\mathbf{g}$ Western blot analysis of cleaved PARP in three representative tumors from each group in $\mathbf{d}$. h The schematic representation of the proposed model for T-DM1 cytotoxicity and sensitization with PLK1 inhibition. SAC-dependent mitotic arrest in T-DM1-treated or T-DM1/volasertib-treated WT and resistant cells, respectively, leads to $\mathrm{CDK} 1$-dependent $\mathrm{Bcl}-2 / \mathrm{xL}$ phosphorylation and inactivation, followed by caspase activation and a caspase-dependent DNA damage, which ultimately results in cell death. $* P<0.05, * * P<0.01$

apoptotic Bcl-2 family members by active cyclin B1/CDK1 complex in prolonged mitosis. Most importantly, PLK1 and $\mathrm{Bcl}-2 / \mathrm{xL}$ overexpression represent two inter-connected resistance mechanisms where inhibition of the former leads to inactivation of the latter both in acquired and in de novo T-DM1 resistance.

\section{Volasertib overcomes T-DM1 resistance in 3D culture and in vivo xenografts}

To validate T-DM1 sensitization by volasertib in vivo, we first generated 3D models of the tumorigenic cell lines in Matrigel. We selected BT-474 as model for the acquired resistance, because SK-BR-3 cells are not tumorigenic. As shown in Fig. 7a, BT-474 T-DM1R cells exhibited loss of T-DM1 response also in 3D. Notably, there was synergistic growth inhibition $(\mathrm{CI}=0.49)$ conferred by the combination of T-DM1 and volasertib in 3D (Fig. 7b), which is in concordance with results obtained in two-dimensional (2D) culture (Fig. 2e). We next tested volasertib-mediated sensitization in de novo T-DM1-resistant JIMT-1 cells grown in 3D. Synergistic growth inhibition $(\mathrm{CI}=0.4)$ was indeed seen when T-DM1 was combined with volasertib in JIMT-1 cells grown in Matrigel (Fig. 7c).

The clinical use of T-DM1 is confined to patients who have disease progression after trastuzumab treatment. The de novo trastuzumab-resistant, HER2-overexpressing breast cancer cell line, JIMT-1, is unique in terms of its trastuzumab resistance mechanisms which have been shown to be multi-factorial compared to other trastuzumab-resistant models [43]. More importantly, we have provided several in vitro evidence that they are also de novo T-DM1 resistant with similar mechanisms that we uncovered in our acquired T-DM1-resistant models (Fig. 6). This cell line is also highly tumorigenic with short latency, and has been used in several studies to develop in vivo HER2-positive breast tumors, especially in the context of trastuzumab resistance [42, 44, 45]. These properties made JIMT-1 a highly suitable model for testing T-DM1/volasertib combination in vivo with high clinical relevance. Combination treatment of tumors with T-DM1 and volasertib at doses that were mostly ineffective when applied individually completely abrogated tumor growth (Fig. 7d). Tumor weights were also significantly less in combination-treated group as compared to individual treatments (Fig. 7e). Hematoxylin and eosin and Ki67 staining demonstrated high levels of necrosis accompanied by a lower proliferation capacity in the combination-treated tumors (Fig. 7f). Furthermore, the apoptotic marker, cleaved PARP, was higher in combination group, further demonstrating apoptosis as the mechanism of cell death with T-DM1/volasertib combination in vivo as well (Fig. 7g). Overall, PLK1 inhibition, when combined with T-DM1, sensitized resistant tumors in vivo, by inhibiting tumor growth.

\section{Discussion}

Effective treatment of T-DM1 refractory breast cancer patients is currently not possible due to lack of functional studies on the molecular mechanisms governing T-DM1 resistance. However, knowledge of such mechanisms would be the basis for the development of combination therapies, thus overcoming T-DM1 resistance and ultimately increasing patient survival [46]. Among the proposed mechanisms of resistance development are poor internalization of the HER2-T-DM1 complex, defective endosomal and lysosomal actions, drug efflux pumps which may export DM1, alterations in tubulins, activation of the cell survival pathways or modulation of the apoptotic signaling $[14,20]$. Here, we utilized a systematic functional approach to decipher the molecular and phenotypic signatures of $\mathrm{T}$ DM1 resistance through combining RNA sequencing, targeted proteomics and a targeted siRNA sensitization screen. This identified PLK1 as a druggable target that is upregulated in T-DM1 resistance and mediates most of the drugassociated resistance processes. PLK1 inhibition with volasertib increased cell death in the presence of T-DM1 by inducing SAC-dependent mitotic arrest, followed by CDK1-dependent phosphorylation and inactivation of Bcl- 
$2 / x L$. Together, this led to the activation of caspases and of caspase-dependent DNA damage (Fig. 7h). Supported with the $3 \mathrm{D}$ culture and in vivo validation of T-DM1 sensitization via volasertib, our results provide valuable insights into the novel mechanisms of T-DM1 resistance.

PLK1 is the most ancestral and well-studied member of the four polo-like kinase family members [47, 48]. It is a Ser/Thr kinase, which plays central roles in many biological processes and performs lots of different functions in a cell cycle-dependent manner, most of which are attributed to mitotic phase [49]. PLK1 is overexpressed in a variety of solid tumors and hematological malignancies both in the context of tumor progression and drug resistance [50-56]; however, its involvement in drug resistance of HER2positive tumors has not been reported before. Cancer cells were shown to be more sensitive to PLK1 inhibition than normal cells making PLK1 a highly attractive drug target in cancer therapy [57]. Volasertib is an ATP-competitive inhibitor of PLK1 and was shown to be effective in various different cancers, in both solid tumors and hematological malignancies [25, 50, 58]. Although it is currently being tested in phase III trials on AML patients, no clinical trial has been initiated thus far in the context of breast cancer. Here, we report a role of PLK1 inhibition with volasertib in re-establishing T-DM1 sensitivity both in vitro and in vivo, which was mainly mediated by regain of the drugassociated phenotypes: mitotic arrest, apoptosis and DNA damage. These data provide pre-clinical evidence for targeting PLK1 in T-DM1 refractory, HER2-positive breast cancer patients.

Bcl-2 is a well-studied anti-apoptotic member of the Bcl2 family proteins, and is central to intrinsic apoptosis [59, $60]$. Expression of Bcl-2 is frequently deregulated in cancer progression and also associated with acquisition of drug resistance [61, 62]. We showed a strong overexpression of $\mathrm{Bcl}-2$ in acquired and Bcl-xL in de novo T-DM1 resistance, thereby protecting cells from apoptotic cell death induced by T-DM1 treatment (Figs. 4f-i and 6d, e). CDK1dependent phosphorylation and inactivation of Bcl-2 in TDM1/volasertib combination-treated resistant cells was preceded by SAC-dependent mitotic arrest, locating Bcl-2 deactivation downstream of PLK1 inhibition as a death executioner, linking prolonged mitosis to apoptosis via CDK1-mediated phosphorylation (Fig. 5e-h). In a recent study, inhibition of PLK1 and Bcl-2 were shown to synergize in a proteasome-dependent manner in MYCoverexpressing glioblastoma [63]. In this system, the antitumor effect of ABT-199, a Bcl-2-specific inhibitor, was enhanced by volasertib which was attributed to activation of the proteasome by PLK1 inhibition which led to degradation of Mcl-1, a compensatory protein of Bcl-2. Here, we have demonstrated a more $\mathrm{Bcl}-2 / \mathrm{xL}$-specific regulation of
PLK1 inhibition with little or no effect on Mcl-1 expression and binding to pro-apoptotic Bim (Fig. 4d). This could be due to the fact that overexpression of $\mathrm{Bcl}-2 / \mathrm{xL}$ in $\mathrm{T}-\mathrm{DM} 1$ resistance makes these proteins preferred targets for regulation as a means to rewire apoptotic signaling. We showed that this regulation is mainly through serine 70 phosphorylation of $\mathrm{Bcl}-2$, thus altering its affinity for binding to an activator $\mathrm{BH} 3$-only protein, Bim. This might free Bim from the inhibitory effect of Bcl-2 so that it can initiate apoptotic signaling (Fig. 4d, e). These data suggest a novel link between Bcl-2-regulated apoptosis and PLK1 through CDK1-dependent phosphorylation in cells that undergo mitotic arrest upon PLK1 inhibition.

The cascade of events initiated upon combination of volasertib with T-DM1 in the resistant models highlighted some proteins/protein complexes as switches mediating transformation between cellular states being provoked by the T-DM1/volasertib combination. Combination therapyinduced mitotic arrest was found to be dependent on the activity of the spindle checkpoint, a well-established gatekeeper of mitotic fidelity [64]. Owing to its ability to halt the cell cycle, inactivation of SAC is frequently associated with cancer progression and resistance against anti-cancer therapies $[65,66]$. Therefore, any treatment that can reactivate the spindle checkpoint in cells under mitotic stress would be beneficial in overcoming resistance against any given therapy. Although PLK1 inhibition has previously been demonstrated to cause SAC activation in a limited number of studies $[67,68]$, it has never been proposed as a way of overcoming resistance against anti-mitotic agents. Prolonged mitosis upon SAC activation is known to cause apoptotic cell death, although the exact mechanisms have not been fully elucidated. Here, we have shown that CDK1dependent Bcl-2 phosphorylation impairs sequestration of the pro-apoptotic $\mathrm{BH} 3$ protein, Bim, thereby linking mitotic arrest to apoptotic cell death. This was further supported by the loss of serine 70 phosphorylation of $\mathrm{Bcl}-2$ and of apoptosis observed upon CDK1 inhibition (Fig. 5g, h). Altogether, our data present a novel mechanism initiated with (1) SAC-dependent mitotic arrest which is accompanied by (2) CDK1-dependent Ser70 phosphorylation and inactivation of Bcl-2, (3) activation of caspases and (4) caspase-dependent DNA damage during T-DM1 sensitization with PLK1 inhibition, which is a complete regain of TDM1 cytotoxicity in sensitive models (Fig. 7h). Most importantly, the identified synergistic growth inhibition by the combination of T-DM1 and volasertib was highly consistent between acquired and de novo resistant models in both 2D and 3D cultures, and was mediated by the same set of events: mitotic arrest, apoptosis and DNA damage. Our findings thus provide strong support for the mechanism 
proposed here to be a common mechanism of T-DM1 resistance.

In summary, we uncovered the molecular and phenotypic landscapes of T-DM1 resistance by an in-depth characterization of two different acquired T-DM1-resistant models, as well as a de novo resistant model, and identified PLK1 as a novel drug target in T-DM1 refractory HER2overexpressing breast cancer. We believe that our results are the first in providing the detailed characterization of $\mathrm{T}$ DM1 cytotoxicity and molecular decoding of the acquired and de novo resistance mechanisms associated with longterm T-DM1 treatment. The strong synergy between volasertib and T-DM1 in the in vivo resistant models in terms of tumor regression holds great promise for this combination to be tested in clinics for the treatment of T-DM1 refractory patients. As volasertib is already being tested in phase III trials, it would be straightforward to perform clinical testing also of combination treatment with T-DM1 and volasertib as this regimen holds the promise to ultimately increase patient survival in aggressive, treatment refractory HER2positive breast cancer.

\section{Materials and methods}

\section{Cell culture and reagents}

Two human HER2-positive breast cancer cell lines BT-474 and SK-BR-3 were obtained from ATCC (Manassas, VA, USA) and de novo trastuzumab/T-DM1-resistant HER2positive breast cancer cell line JIMT-1 was kindly provided by Ali Osmay Gure from Bilkent University, Ankara, Turkey. Cells were cultured in Dulbecco's modified Eagle's medium (Lonza, NJ, USA), supplemented with $10 \%$ fetal bovine serum (Lonza), $50 \mathrm{U} / \mathrm{ml}$ penicillin/streptomycin and $1 \%$ non-essential amino acid. BT-474 cells were also supplemented with $0.1 \%$ insulin. All cell lines were tested for mycoplasma contamination regularly using MycoAlert Mycoplasma Detection Kit (Lonza) and were authenticated by STR sequencing (Promega).

\section{Development of T-DM1-resistant cell lines and cell viability assay}

T-DM1 was kindly provided by Genentech USA Inc. and dissolved in sterile water. Resistant models were developed by culturing BT-474 and SK-BR-3 breast cancer cell lines with increasing doses of T-DM1 continuously over 9 months. Degree of resistance was assessed by performing viability assays in quadruplicates in every 6 weeks with the Cell Titer Glo Cell Viability Kit (Promega, Madison, WI, USA) according to the manufacturer's instructions. Data were analyzed with GraphPad Prism software and were presented as percentage $(\%)$ cell viability relative to vehicletreated control.

Synergy scores for the T-DM1/volasertib combination were calculated by Bliss Independence Model [28] with the following formula:

$\mathrm{CI}=\frac{\mathrm{Ea}+\mathrm{Eb}-\mathrm{Ea} \times \mathrm{Eb}}{\mathrm{Eab}}$, where $\mathrm{Ea}$ and $\mathrm{Eb}$ represent growth inhibition after treatment with the two drugs alone, and Eab represents growth inhibition obtained after treatment with combination of the two drugs.

\section{D culturing of cells}

Cells were grown on top of Matrigel, as previously described [69]. Briefly, culture plates were covered with Matrigel, and cells were seeded on top, in $30 \mu \mathrm{l}$ growth media, with a density of 10,000 and 6000 cells per well for BT-474 and JIMT-1, respectively. After cell attachment for $30 \mathrm{~min}$, treatments were applied in $30 \mu \mathrm{l}$ growth media with 10\% Matrigel (BD Biosciences). Since drug treatment was applied on the same day of seeding, sensitivity was expected to be higher, and therefore we have chosen the doses for T-DM1 and volasertib as being lower than the ones used in 2D experiments. Doses of T-DM1 and volasertib were: $10 \mu \mathrm{g} / \mathrm{ml}$ and $20 \mathrm{nM}$ for BT-474 T-DM1R and $0.5 \mu \mathrm{g} / \mathrm{ml}$ and $20 \mathrm{nM}$ for JIMT-1.

\section{RNA-Seq and data analysis}

RNA sequencing was done for each condition (BT-474 WT and T-DM1R and SK-BR-3 WT and T-DM1R) in duplicates using Illumina HiSeq 2000 at German Cancer Research Center (DKFZ). Around 300 million paired-end $2 \times 125 \mathrm{bp}$ reads were generated and raw sequence data were analyzed by TopHat and Cufflinks algorithms [70]. Details are provided in Supplementary Materials and Methods.

\section{Quantitative RT-PCR analysis}

Total RNA isolation, cDNA generation and quantitative RT-PCR analysis was done in triplicates as previously described [71, 72]. Sequences of the qRT-PCR primers are listed in Supplementary Table S3. For the analysis of data, $\Delta \Delta C_{\mathrm{T}}$ method [73] was used.

\section{Transient transfection with siRNAs and overexpression vectors, and inhibitor treatments}

Transient transfection with siRNAs is listed in Supplementary Table S4, and overexpression vectors and inhibitor treatments are provided in Supplementary Materials and Methods. 


\section{Reverse Phase Protein Arrays}

RPPA experiments were performed as previously described $[74,75]$. Briefly, cell lysates from two biological replicates for each condition were spotted in nitrocellulose-coated glass slides (Oncyte Avid, Grace-Biolabs, Bend, OR, USA) in technical triplicates. All the primary antibodies used were previously validated through Western blots to test their specificity. Signal intensities of spots were quantified using GenePixPro 5.0 (Molecular Devices, Sunnyvale, CA, USA). Details are provided in Supplementary Materials and Methods.

\section{Co-immunoprecipitation}

Proteins were immunoprecipitated with Bim antibody and the co-immunoprecipitates were captured by Dynabeads Protein G. Immunoprecipitates and total extracts were resolved by $10 \%$ sodium dodecyl sulfate polyacrylamide gel electrophoresis (SDS-PAGE), blotted to PVDF membranes and incubated with the antibodies. Details are provided in Supplementary Materials and Methods.

\section{Western blotting}

Protein isolation and Western blotting were done as previously described [72]. List of antibodies was provided in Supplementary Table S5.

All other materials and methods are provided in the Supplementary Materials and Methods.

Acknowledgements This project was supported by TUBITAK-BMBF Bilateral Grant numbers: TUBITAK, $214 Z 130$ (to ÖŞ) and BMBF WTZ, 01DL16003 (to SW). ÖŞ further acknowledges the support from EMBO Installation Grant Number 2791. Special thanks to Genentech, USA Inc. for providing us with T-DM1 under Material Transfer Agreement with MTA number OR-213615. We thank the DKFZ Genomics and Proteomics Core Facility for providing sequencing excellent services. We also thank Deniz Atasoy and Pelin Dilsiz from Istanbul Medipol University for their help with confocal microscopy. ÖK acknowledges support from Baskent University and The Science Academy.

Authors contributions ÖS designed and performed experiments; acquired, analyzed and interpreted data; and prepared the manuscript. SB, ÖA and SDE performed experiments; acquired and analyzed data; UR contributed to in vivo experimental design and data collection; EE and CA performed the transcriptome data analyses; AA performed the immunohistochemical stainings of xenografts and contributed to data interpretation; ÖK performed the co-immunoprecipitation experiments and contributed to data interpretation; SW designed experiments, interpreted data and critically read and edited the manuscript; ÖŞ designed the study, oversaw experiments and data analysis, and prepared the manuscript. All authors reviewed and commented on the manuscript.

\section{Compliance with ethical standards}

Conflict of interest The authors declare that they have no competing interests.

\section{References}

1. Siegel RL, Miller KD, Jemal A. Cancer statistics, 2016. CA Cancer J Clin. 2016;66:7-30.

2. Wang Q, Li SH, Wang H, Xiao Y, Sahin O, Brady SW, et al. Concomitant targeting of tumor cells and induction of T-cell response synergizes to effectively inhibit trastuzumab-resistant breast cancer. Cancer Res. 2012;72:4417-28.

3. Turpin J, Ling C, Crosby EJ, Hartman ZC, Simond AM, Chodosh LA, et al. The ErbB2DeltaEx16 splice variant is a major oncogenic driver in breast cancer that promotes a pro-metastatic tumor microenvironment. Oncogene. 2016;35:6053-64.

4. Stingl J, Caldas C. Molecular heterogeneity of breast carcinomas and the cancer stem cell hypothesis. Nat Rev Cancer. 2007;7:791-9.

5. Henjes F, Bender C, von der Heyde S, Braun L, Mannsperger HA, Schmidt $\mathrm{C}$, et al. Strong EGFR signaling in cell line models of ERBB2-amplified breast cancer attenuates response towards ERBB2-targeting drugs. Oncogenesis. 2012;1:e16

6. Vu T, Claret FX. Trastuzumab: updated mechanisms of action and resistance in breast cancer. Front Oncol. 2012;2:62.

7. Hudis CA. Trastuzumab-mechanism of action and use in clinical practice. N Engl J Med. 2007;357:39-51.

8. Sahin O, Frohlich H, Lobke C, Korf U, Burmester S, Majety M, et al. Modeling ERBB receptor-regulated G1/S transition to find novel targets for de novo trastuzumab resistance. BMC Syst Biol. 2009;3:1.

9. Spector NL, Blackwell KL. Understanding the mechanisms behind trastuzumab therapy for human epidermal growth factor receptor 2-positive breast cancer. J Clin Oncol. 2009;27:5838-47.

10. LoRusso PM, Weiss D, Guardino E, Girish S, Sliwkowski MX. Trastuzumab emtansine: a unique antibody-drug conjugate in development for human epidermal growth factor receptor 2positive cancer. Clin Cancer Res. 2011;17:6437-47.

11. Verma S, Miles D, Gianni L, Krop IE, Welslau M, Baselga J, et al. Trastuzumab emtansine for HER2-positive advanced breast cancer. N Engl J Med. 2012;367:1783-91.

12. Lewis Phillips GD, Li G, Dugger DL, Crocker LM, Parsons KL, Mai E, et al. Targeting HER2-positive breast cancer with trastuzumab-DM1, an antibody-cytotoxic drug conjugate. Cancer Res. 2008;68:9280-90.

13. Barok M, Tanner M, Koninki K, Isola J. Trastuzumab-DM1 causes tumour growth inhibition by mitotic catastrophe in trastuzumab-resistant breast cancer cells in vivo. Breast Cancer Res. 2011;13:R46.

14. Barok M, Joensuu H, Isola J. Trastuzumab emtansine: mechanisms of action and drug resistance. Breast Cancer Res. 2014;16:209.

15. Marusyk A, Almendro V, Polyak K. Intra-tumour heterogeneity: a looking glass for cancer? Nat Rev Cancer. 2012;12:323-34.

16. Roden DM, Wilke RA, Kroemer HK, Stein CM. Pharmacogenomics: the genetics of variable drug responses. Circulation. 2011;123:1661-70.

17. Wright N, Rida PCG, Aneja R. Tackling intra- and inter-tumor heterogeneity to combat triple negative breast cancer. Front Biosci. 2017;22:1549-80.

18. Nicoletti R, Lopez S, Bellone S, Cocco E, Schwab CL, Black JD, et al. T-DM1, a novel antibody-drug conjugate, is highly effective 
against uterine and ovarian carcinosarcomas overexpressing HER2. Clin Exp Metastas-. 2015;32:29-38.

19. Hayashi T, Seiler R, Oo HZ, Jager W, Moskalev I, Awrey S, et al. Targeting HER2 with T-DM1, an antibody cytotoxic drug conjugate, is effective in HER2 over expressing bladder cancer. $\mathbf{J}$ Urol. 2015;194:1120-31.

20. Gagliato DM, Jardim DL, Marchesi MS, Hortobagyi GN. Mechanisms of resistance and sensitivity to anti-HER2 therapies in HER2 + breast cancer. Oncotarget. 2016;7:64431-46.

21. Gajria D, Chandarlapaty S. HER2-amplified breast cancer: mechanisms of trastuzumab resistance and novel targeted therapies. Expert Rev Anticancer Ther. 2011;11:263-75.

22. Oroudjev E, Lopus M, Wilson L, Audette C, Provenzano C, Erickson $\mathrm{H}$, et al. Maytansinoid-antibody conjugates induce mitotic arrest by suppressing microtubule dynamic instability. Mol Cancer Ther. 2010;9:2700-13.

23. Lee KS, Oh DY, Kang YH, Park JE. Self-regulated mechanism of Plk1 localization to kinetochores: lessons from the Plk1-PBIP1 interaction. Cell Div. 2008;3:4.

24. Lindon C, Pines J. Ordered proteolysis in anaphase inactivates Plk1 to contribute to proper mitotic exit in human cells. J Cell Biol. 2004;164:233-41.

25. Yim H. Current clinical trials with polo-like kinase 1 inhibitors in solid tumors. Anticancer Drugs. 2013;24:999-1006.

26. DeAngelo DJ, Sekeres MA, Ottmann OG, Sanz MA, Naoe T, Taube T, et al. Phase III randomized trial of volasertib combined with low-dose cytarabine (LDAC) versus placebo plus LDAC in patients aged $>=65$ years with previously untreated, acute myeloid leukemia (AML) ineligible for intensive remission induction therapy. Clin Lymphoma Myeloma Leuk. 2015;15: S194.

27. Dohner H, Lubbert M, Fiedler W, Fouillard L, Haaland A, Brandwein JM, et al. Randomized, phase 2 trial of low-dose cytarabine with or without volasertib in AML patients not suitable for induction therapy. Blood. 2014;124:1426-33.

28. Mager PP. Structure-toxicity relationships applied to bicyclic organophosphorus poisons. Pharmazie. 1981;36:382-3.

29. Marcotte R, Sayad A, Brown KR, Sanchez-Garcia F, Reimand J, Haider M, et al. Functional genomic landscape of human breast cancer drivers, vulnerabilities, and resistance. Cell. 2016;164:293-309.

30. Daemen A, Griffith OL, Heiser LM, Wang NJ, Enache OM, Sanborn Z, et al. Modeling precision treatment of breast cancer. Genome Biol. 2013;14:R110.

31. Akbani R, Ng PK, Werner HM, Shahmoradgoli M, Zhang F, Ju Z, et al. A pan-cancer proteomic perspective on The Cancer Genome Atlas. Nat Commun. 2014;5:3887.

32. Gluck S, Ross JS, Royce M, McKenna EF Jr., Perou CM, Avisar E, et al. TP53 genomics predict higher clinical and pathologic tumor response in operable early-stage breast cancer treated with docetaxel-capecitabine $+/-$ trastuzumab. Breast Cancer Res Treat. 2012;132:781-91.

33. Scatena CD, Stewart ZA, Mays D, Tang LJ, Keefer CJ, Leach SD, et al. Mitotic phosphorylation of Bcl-2 during normal cell cycle progression and taxol-induced growth arrest. J Biol Chem. 1998;273:30777-84.

34. Yamamoto K, Ichijo H, Korsmeyer SJ. BCL-2 is phosphorylated and inactivated by an ASK1/Jun N-terminal protein kinase pathway normally activated at $\mathrm{G}(2) / \mathrm{M}$. Mol Cell Biol. 1999;19:8469-78.

35. Srivastava RK, Mi QS, Hardwick JM, Longo DL. Deletion of the loop region of Bcl-2 completely blocks paclitaxel-induced apoptosis. Proc Natl Acad Sci USA. 1999;96:3775-80.

36. Pathan N, Aime-Sempe C, Kitada S, Basu A, Haldar S, Reed JC. Microtubule-targeting drugs induce bcl-2 phosphorylation and association with Pin1. Neoplasia. 2001;3:550-9.
37. Tse C, Shoemaker AR, Adickes J, Anderson MG, Chen J, Jin S, et al. ABT-263: a potent and orally bioavailable Bcl-2 family inhibitor. Cancer Res. 2008;68:3421-8.

38. Manchado E, Guillamot M, Malumbres M. Killing cells by targeting mitosis. Cell Death Differ. 2012;19:369-77.

39. Musacchio A, Salmon ED. The spindle-assembly checkpoint in space and time. Nat Rev Mol Cell Biol. 2007;8:379-93.

40. Hain KO, Colin DJ, Rastogi S, Allan LA, Clarke PR. Prolonged mitotic arrest induces a caspase-dependent DNA damage response at telomeres that determines cell survival. Sci Rep. 2016;6:26766.

41. Orth JD, Loewer A, Lahav G, Mitchison TJ. Prolonged mitotic arrest triggers partial activation of apoptosis, resulting in DNA damage and p53 induction. Mol Biol Cell. 2012;23:567-76.

42. Tanner M, Kapanen AI, Junttila T, Raheem O, Grenman S, Elo J, et al. Characterization of a novel cell line established from a patient with Herceptin-resistant breast cancer. Mol Cancer Ther. 2004;3:1585-92.

43. Koninki K, Barok M, Tanner M, Staff S, Pitkanen J, Hemmila P, et al. Multiple molecular mechanisms underlying trastuzumab and lapatinib resistance in JIMT-1 breast cancer cells. Cancer Lett. 2010;294:211-9.

44. Barok M, Isola J, Palyi-Krekk Z, Nagy P, Juhasz I, Vereb G, et al. Trastuzumab causes antibody-dependent cellular cytotoxicitymediated growth inhibition of submacroscopic JIMT-1 breast cancer xenografts despite intrinsic drug resistance. Mol Cancer Ther. 2007;6:2065-72.

45. Leow CC, Chesebrough J, Coffman KT, Fazenbaker CA, Gooya J, Weng D, et al. Antitumor efficacy of IPI-504, a selective heat shock protein 90 inhibitor against human epidermal growth factor receptor 2-positive human xenograft models as a single agent and in combination with trastuzumab or lapatinib. Mol Cancer Ther. 2009;8:2131-41.

46. Recondo G Jr, de la Vega M, Galanternik F, Diaz-Canton E, Leone BA, Leone JP. Novel approaches to target HER2-positive breast cancer: trastuzumab emtansine. Cancer Manag Res. 2016;8:57-65.

47. Zitouni S, Nabais C, Jana SC, Guerrero A, Bettencourt-Dias M. Polo-like kinases: structural variations lead to multiple functions. Nat Rev Mol Cell Biol. 2014;15:433-52.

48. Barr FA, Sillje HH, Nigg EA. Polo-like kinases and the orchestration of cell division. Nat Rev Mol Cell Biol. 2004;5:429-40.

49. van Vugt MA, Medema RH. Getting in and out of mitosis with Polo-like kinase-1. Oncogene. 2005;24:2844-59.

50. Gleixner KV, Ferenc V, Peter B, Gruze A, Meyer RA, Hadzijusufovic E, et al. Polo-like kinase 1 (Plk1) as a novel drug target in chronic myeloid leukemia: overriding imatinib resistance with the Plk1 inhibitor BI 2536. Cancer Res. 2010;70:1513-23.

51. Cheng L, Wang CC, Jing JH. Polo-like kinase 1 as a potential therapeutic target for osteosarcoma. Curr Pharm Des. 2015;21:1347-50.

52. Maire V, Nemati F, Richardson M, Vincent-Salomon A, Tesson B, Rigaill G, et al. Polo-like kinase 1: a potential therapeutic option in combination with conventional chemotherapy for the management of patients with triple-negative breast cancer. Cancer Res. 2013;73:813-23.

53. Spankuch B, Kurunci-Csacsko E, Kaufmann M, Strebhardt K. Rational combinations of siRNAs targeting Plk1 with breast cancer drugs. Oncogene. 2007;26:5793-807.

54. Strebhardt K, Ullrich A. Targeting polo-like kinase 1 for cancer therapy. Nat Rev Cancer. 2006;6:321-30.

55. Degenhardt Y, Lampkin T. Targeting Polo-like kinase in cancer therapy. Clin Cancer Res. 2010;16:384-9.

56. Liu X. Targeting Polo-like kinases: a promising therapeutic approach for cancer treatment. Transl Oncol. 2015;8:185-95.

57. Liu X, Lei M, Erikson RL. Normal cells, but not cancer cells, survive severe Plk1 depletion. Mol Cell Biol. 2006;26:2093-108. 
58. Winkles JA, Alberts GF. Differential regulation of polo-like kinase 1,2,3, and 4 gene expression in mammalian cells and tissues. Oncogene. 2005;24:260-6.

59. Elmore S. Apoptosis: a review of programmed cell death. Toxicol Pathol. 2007;35:495-516.

60. Tait SW, Green DR. Mitochondria and cell death: outer membrane permeabilization and beyond. Nat Rev Mol Cell Biol. 2010;11:621-32.

61. Rantanen S, Monni O, Joensuu H, Franssila K, Knuutila S. Causes and consequences of BCL2 overexpression in diffuse large B-cell lymphoma. Leuk Lymphoma. 2001;42:1089-98.

62. Raffo AJ, Perlman H, Chen MW, Day ML, Streitman JS, Buttyan R. Overexpression of bcl-2 protects prostate cancer cells from apoptosis in vitro and confers resistance to androgen depletion in vivo. Cancer Res. 1995;55:4438-45.

63. Xiao D, Yue M, Su H, Ren P, Jiang J, Li F, et al. Polo-like kinase1 regulates Myc stabilization and activates a feedforward circuit promoting tumor cell survival. Mol Cell. 2016;64:493-506.

64. Kops GJ, Weaver BA, Cleveland DW. On the road to cancer: aneuploidy and the mitotic checkpoint. Nat Rev Cancer. 2005;5:773-85.

65. Vogel C, Kienitz A, Muller R, Bastians H. The mitotic spindle checkpoint is a critical determinant for topoisomerase-based chemotherapy. J Biol Chem. 2005;280:4025-8.

66. Yamada HY, Gorbsky GJ. Spindle checkpoint function and cellular sensitivity to antimitotic drugs. Mol Cancer Ther. 2006;5:2963-9.

67. Trakala M, Partida D, Salazar-Roa M, Maroto M, Wachowicz P, de Carcer G, et al. Activation of the endomitotic spindle assembly checkpoint and thrombocytopenia in Plk1-deficient mice. Blood. 2015;126:1707-14.
68. Yuan J, Sanhaji M, Kramer A, Reindl W, Hofmann M, Kreis NN, et al. Polo-box domain inhibitor poloxin activates the spindle assembly checkpoint and inhibits tumor growth in vivo. Am J Pathol. 2011;179:2091-9.

69. Lee GY, Kenny PA, Lee EH, Bissell MJ. Three-dimensional culture models of normal and malignant breast epithelial cells. Nat Methods. 2007;4:359-65.

70. Trapnell C, Roberts A, Goff L, Pertea G, Kim D, Kelley DR, et al. Differential gene and transcript expression analysis of RNA-seq experiments with TopHat and Cufflinks. Nat Protoc. 2012;7:562-78.

71. Mutlu M, Saatci O, Ansari SA, Yurdusev E, Shehwana H, Konu $\mathrm{O}$, et al. miR-564 acts as a dual inhibitor of PI3K and MAPK signaling networks and inhibits proliferation and invasion in breast cancer. Sci Rep. 2016;6:32541.

72. Raza U, Saatci O, Uhlmann S, Ansari SA, Eyupoglu E, Yurdusev E, et al. The miR-644a/CTBP1/p53 axis suppresses drug resistance by simultaneous inhibition of cell survival and epithelial-mesenchymal transition in breast cancer. Oncotarget. 2016;7:49859-77.

73. Livak KJ, Schmittgen TD. Analysis of relative gene expression data using real-time quantitative PCR and the 2(-Delta Delta C (T)) Method. Methods. 2001;25:402-8.

74. Sahin O, Lobke C, Korf U, Appelhans H, Sultmann H, Poustka A, et al. Combinatorial RNAi for quantitative protein network analysis. Proc Natl Acad Sci USA. 2007;104:6579-84.

75. Sonntag J, Schluter K, Bernhardt S, Korf U. Subtyping of breast cancer using reverse phase protein arrays. Expert Rev Proteom. 2014;11:757-70. 